Supporting Information for

\title{
Synthesis of $\mathbf{2 H}$-Indazoles via Lewis-Acid Promoted Cyclization of 2-(Phenylazo)benzonitriles
}

\author{
Laura D. Shirtcliff, Jazmin Rivers and Michael M. Haley* \\ Department of Chemistry, University of Oregon, Eugene, OR 97403-1253 \\ haley@uoregon.edu
}

Table of Contents

Page

Experimental procedures

$\mathrm{X}$-ray data for $\mathbf{6} \mathbf{j}$

S11

NMR spectra

S20 
Materials and Methods. ${ }^{1} \mathrm{H}$ and ${ }^{13} \mathrm{C}$ NMR spectra were recorded on a $300 \mathrm{MHz}\left({ }^{1} \mathrm{H}, 299.95\right.$ $\mathrm{MHz} ;{ }^{13} \mathrm{C}, 75.43 \mathrm{MHz}$ ) NMR spectrometer. Chemical shifts ( $\delta$ ) are expressed in ppm downfield from $\mathrm{SiMe}_{4}$ using the residual nondeuterated solvent as internal standard $\left(\mathrm{CDCl}_{3}:{ }^{1} \mathrm{H}, 7.26 \mathrm{ppm}\right.$; $\left.{ }^{13} \mathrm{C}, 77.0 \mathrm{ppm} ; \mathrm{C}_{4} \mathrm{D}_{8} \mathrm{O}:{ }^{13} \mathrm{C}, 67.57 \mathrm{ppm}\right)$. Coupling constants are expressed in hertz. IR spectra were recorded using a FTIR 550 spectrometer. Mass spectra were recorded using an LC mass spectrometer with either APCI or ESI sources. Melting points are uncorrected. $\mathrm{CH}_{2} \mathrm{Cl}_{2}$ and hexane were distilled from $\mathrm{CaH}_{2}$ under a nitrogen atmosphere prior to use. THF and $\mathrm{Et}_{2} \mathrm{O}$ were distilled from sodium benzophenone under a nitrogen atmosphere prior to use. All other chemicals were of reagent grade quality and used as obtained from manufacturers. Reactions were carried out in an inert atmosphere (dry $\mathrm{N}_{2}$ or Ar) when necessary. Column chromatography was performed on reagent grade silica gel (230-400 mesh). Preparative and analytical thin-layer chromatography was performed on precoated silica gel plates.

5-tert-Butyl-2-(phenylazo)benzonitrile (5a): Iodide $\mathbf{4 a}^{1}$ (1.13 g, $\left.3.10 \mathrm{mmol}\right)$ and $\mathrm{CuCN}(1.88 \mathrm{~g}$, $21.7 \mathrm{mmol})$ in 1-propanol $(31 \mathrm{~mL})$ were reacted according to general procedure A. Upon concentration, 5c $(0.789 \mathrm{~g}, 98 \%)$ was obtained as a red crystalline solid: $\mathrm{mp} 91.7-92.2{ }^{\circ} \mathrm{C} ;{ }^{1} \mathrm{H}$ NMR $\left(\mathrm{CDCl}_{3}\right) \delta$ 8.04-8.01 (m, 2H), 7.85-7.82 (m, 2H), $7.71(\mathrm{dd}, J=8.7,2.5 \mathrm{~Hz}, 1 \mathrm{H}), 7.56-7.51$ $(\mathrm{m}, 3 \mathrm{H}) 1.38(\mathrm{~s}, 9 \mathrm{H}) ;{ }^{13} \mathrm{C} \mathrm{NMR}\left(\mathrm{CDCl}_{3}\right) \delta 155.1,152.5,151.3,132.4,130.9,130.7,129.4,123.8$, 117.6, 117.0, 113.1, 35.4, 31.1; IR (KBr) 3049, 2961, 2864, 2228, 1595, $1479 \mathrm{~cm}^{-1}$; MS (APCI) $m / z(\%) 264.1\left(\mathrm{M}^{+}+\mathrm{H}, 100\right), 249.1\left(\mathrm{M}^{+}-\mathrm{CH}_{3}, 80\right), 206.0\left(\mathrm{M}^{+}-\mathrm{C}_{4} \mathrm{H}_{9}, 20\right)$.

5-Methyl-2-(phenylazo)benzonitrile (5b): Iodide $\mathbf{4 b}^{1}$ (1.57 g, $\left.4.87 \mathrm{mmol}\right)$ and $\mathrm{CuCN}(2.95 \mathrm{~g}$, $34.1 \mathrm{mmol})$ in 1-propanol $(50 \mathrm{~mL})$ were reacted according to general procedure A. Upon concentration, $\mathbf{5 b}(1.08 \mathrm{~g}, 99 \%)$ was obtained as a red crystalline solid: $\mathrm{mp}$ 94.4-95.1 ${ }^{\circ} \mathrm{C} ;{ }^{1} \mathrm{H}$ NMR $\left(\mathrm{CDCl}_{3}\right) \delta 8.04-8.00(\mathrm{~m}, 2 \mathrm{H}), 7.80(\mathrm{~d}, J=8.1 \mathrm{~Hz}, 1 \mathrm{H}), 7.63(\mathrm{~d}, J=2.7 \mathrm{~Hz}, 1 \mathrm{H}), 7.56-7.47$ $(\mathrm{m}, 4 \mathrm{H}), 2.47(\mathrm{~s}, 3 \mathrm{H}) ;{ }^{13} \mathrm{C} \mathrm{NMR}\left(\mathrm{CDCl}_{3}\right) \delta$ 152.4, 151.3, 142.0, 134.3, 133.8, 132.3, 129.3, 123.7, 117.1, 117.0, 113.3, 21.3; IR (KBr) 3029, 2923, 2853, 2228, 1598, $1480 \mathrm{~cm}^{-1}$; MS (APCI) $m / z(\%) 222.1\left(\mathrm{M}^{+}+\mathrm{H}, 100\right), 195.1\left(\mathrm{M}^{+}-\mathrm{CN}, 15\right), 180.0\left(\mathrm{M}^{+}-\mathrm{C}_{2} \mathrm{H}_{3} \mathrm{~N}, 70\right)$.

2-(Phenylazo)benzonitrile (5c): Iodide $4 \mathbf{c}^{1}$ (1.20 g, $\left.3.89 \mathrm{mmol}\right)$ and $\mathrm{CuCN}(2.36 \mathrm{~g}, 27.3 \mathrm{mmol})$ in 1-propanol $(40 \mathrm{~mL})$ were reacted according to general procedure A. Upon concentration, $\mathbf{5} \mathbf{c}^{2}$ (0.80 g, 99\% yield) was obtained as a red crystalline solid: mp 58.6-59.7 ${ }^{\circ} \mathrm{C} ;{ }^{1} \mathrm{H} \mathrm{NMR}\left(\mathrm{CDCl}_{3}\right)$ ठ 8.06-8.03 (m, 2H), 7.92-7.84 (m, 2H), $7.71(\mathrm{td}, J=7.5,1.5 \mathrm{~Hz}, 1 \mathrm{H}), 7.59-7.54(\mathrm{~m}, 4 \mathrm{H}) ;{ }^{13} \mathrm{C}$ $\mathrm{NMR}\left(\mathrm{CDCl}_{3}\right) \delta 153.4,152.4,133.8,133.6,132.7,131.1,129.5,123.9,117.3,117.1,113.4$; IR 
(KBr) 3033, 2923, 2845, 2223, $1480 \mathrm{~cm}^{-1}$; MS (APCI) $\mathrm{m} / \mathrm{z}(\%) 208.0\left(\mathrm{M}^{+}+\mathrm{H}, 60\right) 180.1\left(\mathrm{M}^{+}-\right.$ $\mathrm{CN}, 100)$.

5-Chloro-2-(phenylazo)benzonitrile (5d): Iodide $\mathbf{4 d}^{1}$ (2.29 g, $\left.6.68 \mathrm{mmol}\right)$ and $\mathrm{CuCN}(4.05 \mathrm{~g}$, $46.8 \mathrm{mmol})$ in 1-propanol $(70 \mathrm{~mL})$ were reacted according to general procedure A. Upon concentration, $5 \mathbf{d}(1.63 \mathrm{~g}, 99 \%)$ was obtained as a red crystalline solid: $\mathrm{mp}$ 93.2-95.0 ${ }^{\circ} \mathrm{C} ;{ }^{1} \mathrm{H}$ NMR $\left(\mathrm{CDCl}_{3}\right) \delta$ 8.04-8.01 (m, 2H), 7.93-7.81 (m, 2H), $7.65(\mathrm{dd}, J=8.7,2.1 \mathrm{~Hz}, 1 \mathrm{H}), 7.57-7.47$ $(\mathrm{m}, 3 \mathrm{H}) ;{ }^{13} \mathrm{C} \mathrm{NMR}\left(\mathrm{CDCl}_{3}\right) \delta 152.3,151.8,137.2,134.0,133.3,133.0,131.5,129.5,124.0$, 118.6, 114.8; IR (KBr) 3077, 2924, 2846, 2232, 1582, $1440 \mathrm{~cm}^{-1}$; MS (APCI) $\mathrm{m} / z$ (\%) 241.9 $\left(\mathrm{M}^{+}+\mathrm{H}, 100\right), 164.1\left(\mathrm{M}^{+}-\mathrm{C}_{6} \mathrm{H}_{5}, 15\right)$.

5-Methoxy-2-(phenylazo)benzonitrile (5e): Iodide $4 \mathrm{e}^{1}$ (1.95 g, $\left.5.77 \mathrm{mmol}\right)$ and $\mathrm{CuCN}$ (3.49 g, $40.4 \mathrm{mmol})$ in 1-propanol $(60 \mathrm{~mL})$ were reacted according to general procedure A. Upon concentration, $5 \mathbf{e}(1.25 \mathrm{~g}, 91 \%)$ was obtained as a red crystalline solid: $\mathrm{mp} 85.3-86.0{ }^{\circ} \mathrm{C} ;{ }^{1} \mathrm{H}$ NMR $\left(\mathrm{CDCl}_{3}\right) \delta$ 8.01-7.98 (m, 2H), $7.91(\mathrm{~d}, J=9.3 \mathrm{~Hz}, 1 \mathrm{H}), 7.56-7.49(\mathrm{~m}, 3 \mathrm{H}), 7.28(\mathrm{~d}, J=2.4$ $\mathrm{Hz}, 1 \mathrm{H}), 7.19(\mathrm{dd}, J=9.3,2.4 \mathrm{~Hz}, 1 \mathrm{H}), 3.92(\mathrm{~s}, 3 \mathrm{H}) ;{ }^{13} \mathrm{C} \mathrm{NMR}\left(\mathrm{CDCl}_{3}\right) \delta$ 161.6, 152.5, 147.7, 132.0, 129.4, 123.6, 120.3, 118.7, 117.2, 117.0, 115.2, 56.2; IR (KBr) 3062, 2938, 2839, 2228, 1599, $1488 \mathrm{~cm}^{-1}$; MS (APCI) $\mathrm{m} / z(\%) 237.8\left(\mathrm{M}^{+}+\mathrm{H}, 100\right) 221.9\left(\mathrm{M}^{+}-\mathrm{CH}_{3}, 100\right) 169.9\left(\mathrm{M}^{+}-\right.$ $\left.\mathrm{C}_{6} \mathrm{H}_{6}, 50\right)$.

2-(4-Bromophenylazo)-5-methoxybenzonitrile (5f): Iodide $4 f^{1}(0.300 \mathrm{~g}, 0.72 \mathrm{mmol})$ and $\mathrm{CuCN}(0.436 \mathrm{~g}, 5.04 \mathrm{mmol})$ in 1-propanol $(7 \mathrm{~mL})$ were reacted according to general procedure A. Upon concentration, $\mathbf{5 f}(0.22 \mathrm{~g}, 99 \%)$ was obtained as a red crystalline solid: mp 144.4-145.1

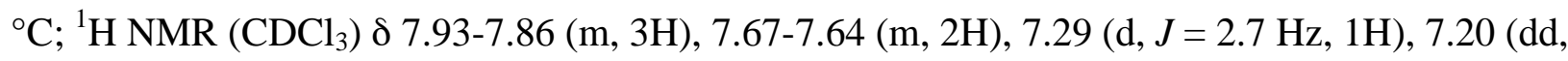
$J=9.0,2.7 \mathrm{~Hz}, 1 \mathrm{H}), 3.93(\mathrm{~s}, 3 \mathrm{H}) ;{ }^{13} \mathrm{C} \mathrm{NMR}\left(\mathrm{CDCl}_{3}\right) \delta 161.9,151.2,147.4,132.7,126.5,125.0$, 120.3, 118.8, 117.3, 115.5, 100.2, 56.3; IR (KBr) 3081, 2975, 2933, 2227, 1593, $1488 \mathrm{~cm}^{-1}$; MS (APCI) $m / z(\%) 315.7\left(\mathrm{M}^{+}+\mathrm{H}, 100\right), 237.0\left(\mathrm{M}^{+}-\mathrm{Br}, 60\right), 160.0\left(\mathrm{M}^{+}-\mathrm{C}_{6} \mathrm{H}_{6} \mathrm{Br}, 70\right)$.

2-(4-Fluorophenylazo)-5-methoxybenzonitrile (5g): Iodide $\mathbf{4 g}^{1}$ (1.84 g, $5.16 \mathrm{mmol}$ ) and $\mathrm{CuCN}$ $(3.13 \mathrm{~g}, 36.1 \mathrm{mmol})$ in 1-propanol $(70 \mathrm{~mL})$ were reacted according to general procedure A. Upon concentration, $\mathbf{5 g}(0.789 \mathrm{~g}, 98 \%)$ was obtained as a red crystalline solid: $\mathrm{mp} 117.5-118.4{ }^{\circ} \mathrm{C} ;{ }^{1} \mathrm{H}$ $\operatorname{NMR}\left(\mathrm{CDCl}_{3}\right) \delta 8.06-8.02(\mathrm{~m}, 2 \mathrm{H}), 7.92(\mathrm{~d}, J=9.0 \mathrm{~Hz}, 1 \mathrm{H}), 7.31(\mathrm{~d}, J=3.0 \mathrm{~Hz}, 1 \mathrm{H}), 7.24-7.20$ $(\mathrm{m}, 3 \mathrm{H}), 3.95(\mathrm{~s}, 3 \mathrm{H}) ;{ }^{13} \mathrm{C} \mathrm{NMR}\left(\mathrm{CDCl}_{3}\right) \delta 166.5,161.4,155.2(\mathrm{~d}, J=1190), 148.8,125.4(\mathrm{~d}, J=$ 8.0 Hz), 120.0, 118.4, 117.0, 116.2 (d, J= 23 Hz), 115.0 56.9; IR (KBr) 3058, 2989, 2223, 1493 $\mathrm{cm}^{-1}$; MS (APCI) m/z (\%) $255.7\left(\mathrm{M}^{+}+\mathrm{H}, 100\right), 240.4\left(\mathrm{M}^{+}-\mathrm{CH}_{3}, 50\right) 159.6\left(\mathrm{M}^{+}-\mathrm{C}_{6} \mathrm{H}_{6} \mathrm{~F}, 45\right)$. 
2-(4-Carbomethoxyphenylazo)-5-methoxybenzonitrile (5h): Iodide $\mathbf{4 h}^{1}$ (0.250 g, $\left.0.631 \mathrm{mmol}\right)$ and $\mathrm{CuCN}(0.384 \mathrm{~g}, 4.42 \mathrm{mmol})$ in 1-propanol $(60 \mathrm{~mL})$ were reacted according to general procedure A. Upon concentration, $5 \mathbf{h}(0.180 \mathrm{~g}, 99 \%)$ was obtained as a red crystalline solid: $\mathrm{mp}$ 210.0-210.4 ${ }^{\circ} \mathrm{C}(\mathrm{dec}) ;{ }^{1} \mathrm{H}$ NMR $\left(\mathrm{CDCl}_{3}\right) \delta 8.18(\mathrm{dt}, J=8.7,1.8 \mathrm{~Hz}, 2 \mathrm{H}), 8.02(\mathrm{dt}, J=8.7,1.8$ $\mathrm{Hz}, 2 \mathrm{H}), 7.93(\mathrm{~d}, J=9.0 \mathrm{~Hz}, 1 \mathrm{H}), 7.31(\mathrm{~d}, J=2.7 \mathrm{~Hz}, 1 \mathrm{H}), 7.21(\mathrm{dd}, J=9.0,2.7 \mathrm{~Hz}, 1 \mathrm{H}), 3.96$ (s, 3H), 3.94 (s, 3H); ${ }^{13} \mathrm{C}$ NMR $\left(\mathrm{CDCl}_{3}\right) \delta 166.6,162.3,154.9,147.5,132.7,130.9,123.4,120.3$, 118.9, 117.5, 116.8, 115.9, 56.3, 52.6; IR (KBr) 3021, 2841, 2227, 1721, 1592, $1343 \mathrm{~cm}^{-1}$; MS (APCI) $m / z$ (\%) $296.1\left(\mathrm{M}^{+}+\mathrm{H}, 100\right), 264.0\left(\mathrm{M}^{+}-\mathrm{CH}_{3} \mathrm{O}, 25\right), 160.0\left(\mathrm{M}^{+}-\mathrm{C}_{8} \mathrm{H}_{7} \mathrm{O}_{2}\right.$

2-(4-Cyanophenylazo)-5-methoxybenzonitrile (5i): Iodide $4 \mathbf{i}^{1}(0.25 \mathrm{~g}, 0.688 \mathrm{mmol})$ and $\mathrm{CuCN}$ $(0.417 \mathrm{~g}, 4.82 \mathrm{mmol})$ in 1-propanol $(20 \mathrm{~mL})$ were reacted according to general procedure A. Upon concentration, $\mathbf{5 i}(0.167 \mathrm{~g}, 92 \%)$ was obtained as a red crystalline solid: $\mathrm{mp}$ 223.3-224.0 ${ }^{\circ} \mathrm{C}(\mathrm{dec}) ;{ }^{1} \mathrm{H}$ NMR $\left(\mathrm{CDCl}_{3}\right) \delta 8.07(\mathrm{dt}, J=8.4,1.8 \mathrm{~Hz}, 2 \mathrm{H}), 7.94(\mathrm{~d}, J=9.0 \mathrm{~Hz}, 1 \mathrm{H}), 7.82(\mathrm{dt}, J$ $=8.4,1.8 \mathrm{~Hz}, 2 \mathrm{H}), 7.33(\mathrm{~d}, J=2.4 \mathrm{~Hz}, 1 \mathrm{H}), 7.23(\mathrm{dd}, J=9.0,2.4 \mathrm{~Hz}, 1 \mathrm{H}), 3.95(\mathrm{~s}, 3 \mathrm{H}) ;{ }^{13} \mathrm{C}$ NMR $\left(\mathrm{CDCl}_{3}\right) \delta 162.6,154.2,147.1,133.5,124.0,120.3,118.8,118.6,117.6,116.3,114.8$, 56.4; IR (KBr) 3098, 3041, 2844, 2223, 1595, $1489 \mathrm{~cm}^{-1}$; MS (APCI) $\mathrm{m} / z(\%) 263.1\left(\mathrm{M}^{+}+\mathrm{H}\right.$, 100).

5-Methoxy-2-(4-nitrophenylazo)benzonitrile (5j): Iodide $\mathbf{4 j}^{1}(0.100 \mathrm{~g}, 0.261 \mathrm{mmol})$ and $\mathrm{CuCN}$ $(0.158 \mathrm{~g}, 1.83 \mathrm{mmol})$ in 1-propanol $(31 \mathrm{~mL})$ were reacted according to general procedure A. Upon concentration, 5j (72.5 mg, 99\%) was obtained as a red crystalline solid: mp 188.7-189.2 ${ }^{\circ} \mathrm{C} ;{ }^{1} \mathrm{H} \mathrm{NMR}\left(\mathrm{CDCl}_{3}\right) \delta 8.38(\mathrm{dt}, J=9.0,1.8 \mathrm{~Hz}, 2 \mathrm{H}), 8.11(\mathrm{dt}, J=9.0,1.8 \mathrm{~Hz}, 2 \mathrm{H}), 7.95(\mathrm{~d}, J=$ $9.0 \mathrm{~Hz}, 1 \mathrm{H}), 7.33(\mathrm{~d}, J=3.0 \mathrm{~Hz}, 1 \mathrm{H}), 7.23(\mathrm{dd}, J=9.0,2.7 \mathrm{~Hz}, 1 \mathrm{H}), 3.96(\mathrm{~s}, 3 \mathrm{H}) ;{ }^{13} \mathrm{C} \mathrm{NMR}$ $\left(\mathrm{CDCl}_{3}\right) \delta 162.8,155.4,149.3,147.2,125.0,124.1,122.4,120.3,118.9,117.7,116.5,56.4$; IR (KBr) 3105, 2925, 2846, 2232, 1596, 1516, $1351 \mathrm{~cm}^{-1}$; MS (APCI) m/z (\%) $283.9\left(\mathrm{M}^{+}+\mathrm{H}, 100\right)$.

Isoindazole imine 6a: Diazene $5 \mathbf{a}(75 \mathrm{mg}, 0.280 \mathrm{mmol})$, alkene $(0.34 \mathrm{~mL}, 2.8 \mathrm{mmol})$, and $\mathrm{BF}_{3} \bullet \mathrm{OEt}_{2}(0.18 \mathrm{~mL}, 1.40 \mathrm{mmol})$ in $\mathrm{CH}_{2} \mathrm{Cl}_{2}(20 \mathrm{~mL})$ were reacted according to general procedure B. After aqueous work-up 6a (92 mg, 94\%) was obtained as a light tan solid: mp 106.5-107.3 ${ }^{\circ} \mathrm{C}$; ${ }^{1} \mathrm{H}$ NMR $\left(\mathrm{CDCl}_{3}\right) \delta$ 7.75-7.71 (m, 2H), $7.59(\mathrm{dd}, J=9.0,1.8,1 \mathrm{H}), 7.47-7.39(\mathrm{~m}, 3 \mathrm{H}), 7.33(\mathrm{tt}, J$ $=7.8,1.5 \mathrm{~Hz}, 1 \mathrm{H}), 7.07(\mathrm{~d}, J=1.8 \mathrm{~Hz}, 1 \mathrm{H}), 1.88(\mathrm{~s}, 3 \mathrm{H}), 1.36(\mathrm{~s}, 9 \mathrm{H}), 1.27(\mathrm{~s}, 9 \mathrm{H}) ;{ }^{13} \mathrm{C} \mathrm{NMR}$ $\left(\mathrm{CDCl}_{3}\right) \delta 185.0,147.4,143.4,140.8,139.8,128.6,127.3,126.9,124.1,117.1,113.7,111.7$, 41.3, 34.7, 31.1, 27.9, 17.9; IR (KBr) 2946, 2360, 1645, $1360 \mathrm{~cm}^{-1}$; MS (APCI) $\mathrm{m} / z$ (\%) 348.3 $\left(\mathrm{M}^{+}+\mathrm{H}, 80\right), 332.2\left(\mathrm{M}^{+}-\mathrm{CH}_{3}, 30\right), 276.2\left(\mathrm{M}^{+}-\mathrm{C}_{6} \mathrm{H}_{10}, 45\right)$. 
Isoindazole imine 6b: Diazene $5 \mathbf{b}(100 \mathrm{mg}, 0.450 \mathrm{mmol})$, alkene $(0.53 \mathrm{~mL}, 4.5 \mathrm{mmol})$, and $\mathrm{BF}_{3} \bullet \mathrm{OEt}_{2}(0.29 \mathrm{~mL}, 2.25 \mathrm{mmol})$ in $\mathrm{CH}_{2} \mathrm{Cl}_{2}(20 \mathrm{~mL})$ were reacted according to general procedure B. After aqueous work-up $6 \mathbf{b}(0.123 \mathrm{~g}, 90 \%)$ was obtained as a light tan solid: $\mathrm{mp} 71.5-72.6{ }^{\circ} \mathrm{C}$; ${ }^{1} \mathrm{H}$ NMR $\left(\mathrm{CDCl}_{3}\right) \delta 7.73(\mathrm{~d}, J=8.5 \mathrm{~Hz}, 2 \mathrm{H}), 7.55(\mathrm{~d}, J=8.5 \mathrm{~Hz}, 1 \mathrm{H}), 7.44(\mathrm{t}, J=8.5 \mathrm{~Hz}, 2 \mathrm{H})$, $7.33(\mathrm{t}, J=8.5 \mathrm{~Hz}, 1 \mathrm{H}), 7.12(\mathrm{dd}, J=8.5,1.5 \mathrm{~Hz}, 1 \mathrm{H}), 6.95(\mathrm{~d}, J=1.5 \mathrm{~Hz}, 1 \mathrm{H}), 2.40(\mathrm{~s}, 3 \mathrm{H})$, 1.89 (s, 3H), 1.27 (s, 9H); ${ }^{13} \mathrm{C} \mathrm{NMR}\left(\mathrm{CDCl}_{3}\right) \delta$ 185.1, 147.4, 140.1, 139.7, 130.0, 128.6, 127.3, 124.1, 117.9, 117.3, 112.1, 41.3, 27.9, 21.8, 17.9; IR (KBr) 3094, 2971, 1641, 1598, $1499 \mathrm{~cm}^{-1}$; MS (APCI) $m / z(\%) 306.2\left(\mathrm{M}^{+}+\mathrm{H}, 100\right), 290.1\left(\mathrm{M}^{+}-\mathrm{CH}_{3}, 20\right), 207.0\left(\mathrm{M}^{+}-\mathrm{C}_{6} \mathrm{H}_{12} \mathrm{~N}, 40\right)$. Isolated also was $7 \mathbf{b}(5 \mathrm{mg}, 5 \%)$ as a light tan solid; spectroscopic details outlined below.

Isoindazole imine 6c: Diazene 5c $(80 \mathrm{mg}, 0.390 \mathrm{mmol})$, alkene $(0.46 \mathrm{~mL}, 3.90 \mathrm{mmol})$, and $\mathrm{BF}_{3} \bullet \mathrm{OEt}_{2}(0.24 \mathrm{~mL}, 1.93 \mathrm{mmol})$ in $\mathrm{CH}_{2} \mathrm{Cl}_{2}(20 \mathrm{~mL})$ were reacted according to general procedure B. After aqueous work-up 6c (95 mg, 84\%) was obtained as a light tan solid: $\mathrm{mp} 83.5-84.9^{\circ} \mathrm{C}$; ${ }^{1} \mathrm{H}$ NMR $\left(\mathrm{CDCl}_{3}\right) \delta 7.78(\mathrm{dt}, J=8.1,1.5 \mathrm{~Hz}, 2 \mathrm{H}), 7.66(\mathrm{~d}, J=8.7 \mathrm{~Hz}, 1), 7.47(\mathrm{tt}, J=8.1,1.5$ $\mathrm{Hz}, 2 \mathrm{H}), 7.39-7.24$ (m, 3H), 7.00 (dd, $J=8.7,1.8 \mathrm{~Hz}, 1 \mathrm{H}), 1.91$ (s, 3H), $1.28(\mathrm{~s}, 9 \mathrm{H})$; ${ }^{13} \mathrm{C}$ NMR $\left(\mathrm{CDCl}_{3}\right) \delta 185.5,148.4,141.0,139.6,128.6,127.5,127.0,124.2,120.6,119.9,117.5,112.0$, 41.3, 27.8, 17.9; IR (KBr) 2966, 2917, 1641, 1597, $1499 \mathrm{~cm}^{-1}$; MS (APCI) $\mathrm{m} / z$ (\%) 292.1 $\left(\mathrm{M}^{+}+\mathrm{H}, 100\right), 193.1\left(\mathrm{M}^{+}-\mathrm{C}_{6} \mathrm{H}_{12} \mathrm{~N}, 15\right)$. Isolated also was 7c (13 mg, 16\%) as a light tan solid; spectroscopic details outlined below.

Isoindazole imine 6d: Diazene $5 \mathbf{d}(100 \mathrm{mg}, 0.410 \mathrm{mmol})$, alkene $(0.49 \mathrm{~mL}, 4.1 \mathrm{mmol})$, and $\mathrm{BF}_{3} \bullet \mathrm{OEt}_{2}(0.26 \mathrm{~mL}, 2.05 \mathrm{mmol})$ in $\mathrm{CH}_{2} \mathrm{Cl}_{2}(20 \mathrm{~mL})$ were reacted according to general procedure B. After aqueous work-up 6d $(0.127 \mathrm{~g}, 95 \%)$ was obtained as a light tan solid: $\mathrm{mp}$ 84.2-85.4 ${ }^{\circ} \mathrm{C}$; ${ }^{1} \mathrm{H} \mathrm{NMR}\left(\mathrm{CDCl}_{3}\right) \delta 7.71(\mathrm{dt}, J=8.4,1.5 \mathrm{~Hz}, 2 \mathrm{H}), 7.57(\mathrm{~d}, J=9.6 \mathrm{~Hz}), 7.45(\mathrm{dt}, J=8.4,1.5 \mathrm{~Hz}$, 2H), $7.36(\mathrm{tt}, J=8.4,1.5 \mathrm{~Hz}, 2 \mathrm{H}), 7.22-7.18(\mathrm{~m}, 2 \mathrm{H}), 1.87(\mathrm{~s}, 3 \mathrm{H}), 1.25(\mathrm{~s}, 9 \mathrm{H}) ;{ }^{13} \mathrm{C} \mathrm{NMR}$ $\left(\mathrm{CDCl}_{3}\right) \delta 186.3,146.8,140.7,139.3,128.7,128.4,127.8,125.9,124.1,119.2,118.6,112.3$, 41.4, 27.8, 18.1; IR (KBr) 3057, 2960, 1644, 1502, $1324 \mathrm{~cm}^{-1}$; MS (APCI) $m / z$ (\%) 326.1 $\left(\mathrm{M}^{+}+\mathrm{H}, 100\right), 227.1\left(\mathrm{M}^{+}-\mathrm{C}_{6} \mathrm{H}_{12} \mathrm{~N}, 60\right)$.

Isoindazole imine 6e: Diazene 5 e $(100 \mathrm{mg}, 0.420 \mathrm{mmol})$, alkene $(0.50 \mathrm{~mL}, 4.2 \mathrm{mmol})$, and $\mathrm{BF}_{3} \cdot \mathrm{OEt}_{2}(0.27 \mathrm{~mL}, 2.05 \mathrm{mmol})$ in $\mathrm{CH}_{2} \mathrm{Cl}_{2}(20 \mathrm{~mL})$ were reacted according to general procedure B. After aqueous work-up the imine (97 mg, 72\%) was obtained as a light tan solid: $\mathrm{mp}$ 123.9$125.0{ }^{\circ} \mathrm{C} ;{ }^{1} \mathrm{H} \mathrm{NMR}\left(\mathrm{CDCl}_{3}\right) \delta 7.72(\mathrm{dt}, J=8.4,1.5 \mathrm{~Hz}, 2 \mathrm{H}), 7.56(\mathrm{~d}, J=9.6 \mathrm{~Hz}, 1 \mathrm{H}), 7.46-7.40$ $(\mathrm{m}, 2 \mathrm{H}) 7.31(\mathrm{tt}, J=8.4,1.5 \mathrm{~Hz}, 2 \mathrm{H}), 7.00(\mathrm{dd}, J=9.6,2.7 \mathrm{~Hz}, 1 \mathrm{H}), 6.37(\mathrm{~d}, J=2.7 \mathrm{~Hz}, 1 \mathrm{H})$; 
${ }^{13} \mathrm{C} \mathrm{NMR}\left(\mathrm{CDCl}_{3}\right) \delta 184.7,154.4,145.4,140.0,139.8,128.6,127.2,124.0,121.9,118.1,111.4$, 96.2, 55.4, 41.3, 27.9, 17.8; IR (KBr) 3040, 2855, 1635, $1518 \mathrm{~cm}^{-1}$; MS (APCI) $\mathrm{m} / z$ (\%) 322.1 $\left(\mathrm{M}^{+}+\mathrm{H}, 100\right), 223.1\left(\mathrm{M}^{+}-\mathrm{C}_{6} \mathrm{H}_{12} \mathrm{~N}, 50\right)$. Isolated also was $7 \mathrm{e}(27 \mathrm{mg}, 27 \%)$ as a light tan solid; spectroscopic details outlined below.

Isoindazole imine 6f: Diazene $\mathbf{5 f}(100 \mathrm{mg}, 0.320 \mathrm{mmol})$, alkene $(0.38 \mathrm{~mL}, 3.20 \mathrm{mmol})$, and $\mathrm{BF}_{3} \bullet \mathrm{OEt}_{2}(0.20 \mathrm{~mL}, 1.60 \mathrm{mmol})$ in $\mathrm{CH}_{2} \mathrm{Cl}_{2}(20 \mathrm{~mL})$ were reacted according to general procedure B. After aqueous work-up $6 \mathbf{f}(0.101 \mathrm{~g}, 79 \%)$ was obtained as a light tan solid: mp 137.5-138.2 ${ }^{\circ} \mathrm{C} ;{ }^{1} \mathrm{H}$ NMR $\left(\mathrm{CDCl}_{3}\right) \delta$ 7.68-7.65 (m, 2H), 7.56-7.52 (m, 3H), $7.00(\mathrm{dd}, J=9.5,2.0 \mathrm{~Hz}, 1 \mathrm{H})$, $6.32(\mathrm{~d}, J=2.0 \mathrm{~Hz}, 1 \mathrm{H}), 3.80(\mathrm{~s}, 3 \mathrm{H}), 1.90(\mathrm{~s}, 3 \mathrm{H}), 1.28(\mathrm{~s}, 9 \mathrm{H}) ;{ }^{13} \mathrm{C} \mathrm{NMR}\left(\mathrm{CDCl}_{3}\right) \delta 184.9$, 154.6, 145.5, 140.0, 138.9, 131.7, 125.3, 122.2, 120.8, 119.1, 111.3, 96.0, 55.4, 41.3, 27.9, 17.9; IR (KBr) 3342, 2964, 1635, $1517 \mathrm{~cm}^{-1}$; MS (APCI) $m / z$ (\%) 400.1 ( $\left.\mathrm{M}^{+}+\mathrm{H}, 100\right), 320.1\left(\mathrm{M}^{+}-\mathrm{Br}\right.$, 15), $301.0\left(\mathrm{M}^{+}-\mathrm{C}_{6} \mathrm{H}_{12} \mathrm{~N}, 15\right)$. Isolated also was $7 \mathbf{f}(10 \mathrm{mg}, 9 \%)$ as a light tan solid; spectroscopic details outlined below.

Isoindazole imine 6g: Diazene 6g (100 mg, $0.390 \mathrm{mmol})$, alkene $(0.46 \mathrm{~mL}, 3.90 \mathrm{mmol})$, and $\mathrm{BF}_{3} \bullet \mathrm{OEt}_{2}(0.25 \mathrm{~mL}, 1.45 \mathrm{mmol})$ in $\mathrm{CH}_{2} \mathrm{Cl}_{2}(20 \mathrm{~mL})$ were reacted according to general procedure B. After aqueous work-up $6 \mathbf{g}(90 \mathrm{mg}, 70 \%)$ was obtained as a light tan solid: $\mathrm{mp} \mathrm{123.4-125.0}{ }^{\circ} \mathrm{C}$; ${ }^{1} \mathrm{H} \mathrm{NMR}\left(\mathrm{CDCl}_{3}\right) \delta 7.70(\mathrm{tt}, J=8.4,2.7 \mathrm{~Hz}, 2 \mathrm{H}), 7.55(\mathrm{dd}, J=9.3 \mathrm{~Hz}, 1 \mathrm{H}), 7.12(\mathrm{tt}, J=8.4,2.7$ Hz, 2H), 7.02 (dd, $J=9.3,2.4 \mathrm{~Hz}, 1 \mathrm{H}), 6.35$ (d, $J=2.4 \mathrm{~Hz}, 1 \mathrm{H}), 3.81$ (s, 3H), 1.90 (s, 3H), 1.26 $(\mathrm{s}, 9 \mathrm{H}) ;{ }^{13} \mathrm{C} \mathrm{NMR}\left(\mathrm{CDCl}_{3}\right) \delta 184.9,160.5,154.5,149.2(\mathrm{~d}, J=1995 \mathrm{~Hz}), 145.4,140.0,125.7$ (d, $J=11.6 \mathrm{~Hz}), 122.0,119.1(\mathrm{~d}, J=11.0 \mathrm{~Hz}), 115.6,111.2,96.1,55.4,41.3,27.9,17.8$; IR $(\mathrm{KBr})$ 2974, 1635, 1519, $1469 \mathrm{~cm}^{-1}$; MS (APCI) $m / z(\%) 340.1\left(\mathrm{M}^{+}+\mathrm{H}, 100\right) 241.1\left(\mathrm{M}^{+}-\mathrm{C}_{6} \mathrm{H}_{12} \mathrm{~N}, 15\right)$. Isolated also was $\mathbf{7 g}$ (19 mg, 25\%) as a light tan solid; spectroscopic details outlined below. Isoindazole imine 6h: Diazene $6 \mathbf{h}(80 \mathrm{mg}, 0.270 \mathrm{mmol})$, alkene $(0.32 \mathrm{~mL}, 2.70 \mathrm{mmol})$, and $\mathrm{BF}_{3} \bullet \mathrm{OEt}_{2}(0.17 \mathrm{~mL}, 1.35 \mathrm{mmol})$ in $\mathrm{CH}_{2} \mathrm{Cl}_{2}(20 \mathrm{~mL})$ were reacted according to general procedure B. After aqueous work-up $6 \mathbf{h}$ (91 mg, 89\%) was obtained as a light yellow solid: mp 119.6-121.1 ${ }^{\circ} \mathrm{C} ;{ }^{1} \mathrm{H}$ NMR $\left(\mathrm{CDCl}_{3}\right) \delta 8.12(\mathrm{dt}, J=8.5,1.8 \mathrm{~Hz}, 2 \mathrm{H}), 7.80(\mathrm{dt}, J=8.5,1.8 \mathrm{~Hz}, 2 \mathrm{H}), 7.54(\mathrm{~d}, J=$ $9.5 \mathrm{~Hz}, 1 \mathrm{H}), 7.01(\mathrm{dd}, J=9.5,2.5 \mathrm{~Hz}, 1 \mathrm{H}), 6.31(\mathrm{~d}, J=2.5 \mathrm{~Hz}, 1 \mathrm{H}), 3.97(\mathrm{~s}, 3 \mathrm{H}), 3.81(\mathrm{~s}, 3 \mathrm{H})$, $1.91(\mathrm{~s}, 3 \mathrm{H}), 1.29(\mathrm{~s}, 9 \mathrm{H}) ;{ }^{13} \mathrm{C} \mathrm{NMR}\left(\mathrm{CDCl}_{3}\right) \delta 185.1,166.4,154.7,145.9,143.53,140.4,130.2$, 128.4, 123.2, 122.7, 119.3, 111.4, 95.9, 55.4, 52.2, 41.4, 27.9, 17.9; IR (KBr) 2996, 1714, 1637, 1519, $1274 \mathrm{~cm}^{-1}$; MS (APCI) $\mathrm{m} / z(\%) 380.2\left(\mathrm{M}^{+}+\mathrm{H}, 100\right), 281.1\left(\mathrm{M}^{+}-\mathrm{C}_{6} \mathrm{H}_{12} \mathrm{~N}, 15\right)$. Isolated also was $7 \mathbf{h}(3 \mathrm{mg}, 4 \%)$ as a light tan solid; spectroscopic details outlined below. 
Isoindazole imine 6i: Diazene $5 \mathbf{i}(80 \mathrm{mg}, 0.30 \mathrm{mmol})$, alkene $(0.35 \mathrm{~mL}, 3.00 \mathrm{mmol})$, and $\mathrm{BF}_{3} \bullet \mathrm{OEt}_{2}(0.19 \mathrm{~mL}, 1.50 \mathrm{mmol})$ in $\mathrm{CH}_{2} \mathrm{Cl}_{2}(20 \mathrm{~mL})$ were reacted according to general procedure B. After aqueous work-up 6i (65 mg, 63\%) was obtained as a yellow solid: $\mathrm{mp} 136.8-138.2{ }^{\circ} \mathrm{C}$; ${ }^{1} \mathrm{H} \mathrm{NMR}\left(\mathrm{CDCl}_{3}\right) \delta 8.03(\mathrm{dt}, J=8.5,1.8 \mathrm{~Hz}, 2 \mathrm{H}), 7.74(\mathrm{dt}, J=8.5,1.8 \mathrm{~Hz}, 2 \mathrm{H}), 7.53(\mathrm{~d}, J=9.5$ $\mathrm{Hz}, 1 \mathrm{H}), 7.02$ (dd, $J=9.5,2.5 \mathrm{~Hz}, 1 \mathrm{H}), 6.26(\mathrm{~d}, J=2.5 \mathrm{~Hz}, 1 \mathrm{H}), 3.80(\mathrm{~s}, 3 \mathrm{H}), 1.94(\mathrm{~s}, 3 \mathrm{H}), 1.31$ $(\mathrm{s}, 9 \mathrm{H}) ;{ }^{13} \mathrm{C} \mathrm{NMR}\left(\mathrm{CDCl}_{3}\right) \delta 185.3,154.9,146.2,143.4,140.5,132.7,123.7,123.2,119.3,118.4$, 111.4, 110.2, 95.7, 55.3, 41.5, 28.0, 18.0; IR (KBr) 2965, 2928, 2227, 1638, 1604, $1525 \mathrm{~cm}^{-1}$; MS (APCI) $m / z(\%) 347.2\left(\mathrm{M}^{+}+\mathrm{H}, 100\right), 248.1\left(\mathrm{M}^{+}-\mathrm{C}_{6} \mathrm{H}_{12} \mathrm{~N}, 15\right)$. Isolated also was $7 \mathbf{i}(24 \mathrm{mg}$, $30 \%$ ) as a light tan solid; spectroscopic details outlined below.

Isoindazole imine 6j: Diazene $5 \mathbf{j}$ (75 mg, $0.270 \mathrm{mmol})$, alkene $(0.46 \mathrm{~mL}, 3.90 \mathrm{mmol})$, and $\mathrm{BF}_{3} \cdot \mathrm{OEt}_{2}(0.31 \mathrm{~mL}, 2.66 \mathrm{mmol})$ in $\mathrm{CH}_{2} \mathrm{Cl}_{2}(20 \mathrm{~mL})$ were reacted according to general procedure B. After aqueous work-up $6 \mathbf{j}$ (76 mg, 78\%) was obtained as a yellow solid: $119.7-120.8{ }^{\circ} \mathrm{C} ;{ }^{1} \mathrm{H}$ $\operatorname{NMR}\left(\mathrm{CDCl}_{3}\right) \delta 8.32(\mathrm{dt}, J=8.5,1.8 \mathrm{~Hz}, 2 \mathrm{H}), 8.11(\mathrm{dt}, J=8.5,1.8 \mathrm{~Hz}, 2 \mathrm{H}), 7.54(\mathrm{~d}, J=9.5 \mathrm{~Hz}$, $1 \mathrm{H}), 7.02(\mathrm{dd}, J=9.5,1.5 \mathrm{~Hz}, 1 \mathrm{H}), 6.26$ (d, $J=1.5 \mathrm{~Hz}, 1 \mathrm{H}), 3.81(\mathrm{~s}, 3 \mathrm{H}), 1.97$ (s, 3H), 1.32 (s, 9H); ${ }^{13} \mathrm{C} \mathrm{NMR}\left(\mathrm{CDCl}_{3}\right) \delta 185.5,155.1,146.3,145.7,145.0,140.7,124.3,123.4,119.4,111.5$, 95.7, 55.3, 41.5, 28.0, 18.0; IR (KBr) 2958, 2850, 1625, 1506, $1322 \mathrm{~cm}^{-1}$; MS (APCI) $\mathrm{m} / z(\%)$ $367.1\left(\mathrm{M}^{+}+\mathrm{H}, 100\right), 268.1\left(\mathrm{M}^{+}-\mathrm{C}_{6} \mathrm{H}_{12} \mathrm{~N}, 15\right)$. Isolated also was $7 \mathbf{j}(17 \mathrm{mg}, 22 \%)$ as a light tan solid; spectroscopic details outlined below.

3-Amino-5-tert-butyl-2-phenyl-2H-indazole (7a): Diazene 5a (75 mg, $0.285 \mathrm{mmol})$ and $\mathrm{SnCl}_{2} \cdot \mathrm{H}_{2} \mathrm{O}(0.270 \mathrm{~g}, 1.43 \mathrm{mmol})$ in $\mathrm{EtOH}(30 \mathrm{~mL})$ were reacted according to general procedure C. Upon concentration, $7 \mathbf{a}(66 \mathrm{mg}, 87 \%)$ was obtained as a tan solid: $\mathrm{mp} 182.1-183.4{ }^{\circ} \mathrm{C} ;{ }^{1} \mathrm{H}$ $\operatorname{NMR}\left(\mathrm{CDCl}_{3}\right) \delta 7.70-7.68(\mathrm{~m}, 2 \mathrm{H}), 7.59-7.54(\mathrm{~m}, 2 \mathrm{H}), 7.51(\mathrm{~d}, J=9.0 \mathrm{~Hz}, 1 \mathrm{H}), 7.46(\mathrm{t}, J=7.5$ $\mathrm{Hz}, 1 \mathrm{H}), 7.42(\mathrm{dd}, J=9.0,1.5 \mathrm{~Hz}, 1 \mathrm{H}), 7.38(\mathrm{~d}, J=1.5 \mathrm{~Hz}), 4.33($ br s, $2 \mathrm{H}), 1.37(\mathrm{~s}, 9 \mathrm{H}) ;{ }^{13} \mathrm{C}$ NMR $\left(\mathrm{CDCl}_{3}\right) \delta 147.3,141.4,138.6,138.0,129.6,128.3,127.4,124.9,116.5,113.1,109.2$, 34.7, 31.2; IR (KBr) 3378, 3134, 1643, $1523 \mathrm{~cm}^{-1}$; MS (APCI) $m / z(\%) 266.1\left(\mathrm{M}^{+}+\mathrm{H}, 100\right)$, $249.1\left(\mathrm{M}^{+}-\mathrm{NH}_{2}, 50\right)$.

3-Amino-5-methyl-2-phenyl-2H-indazole (7b): Diazene $5 \mathbf{b}$ (75 mg, $0.339 \mathrm{mmol})$ and $\mathrm{SnCl}_{2} \bullet \mathrm{H}_{2} \mathrm{O}(0.343 \mathrm{~g}, 1.81 \mathrm{mmol})$ in $\mathrm{EtOH}(30 \mathrm{~mL})$ were reacted according to general procedure C. Upon concentration, $7 \mathbf{b}(66 \mathrm{mg}, 89 \%)$ was obtained as a tan solid: $\mathrm{mp} 174.6-175.5^{\circ} \mathrm{C} ;{ }^{1} \mathrm{H}$ $\operatorname{NMR}\left(\mathrm{CDCl}_{3}\right) \delta 7.68(\mathrm{dt}, J=8.4,2.1 \mathrm{~Hz}, 2 \mathrm{H}), 7.54(\mathrm{tt}, J=8.4,2.1 \mathrm{~Hz}, 2 \mathrm{H}), 7.46-7.41(\mathrm{~m}, 2 \mathrm{H})$ $7.21(\mathrm{~d}, J=1.5 \mathrm{~Hz}, 1 \mathrm{H}), 7.10(\mathrm{dd}, J=9.0,1.5 \mathrm{~Hz}), 4.24(\mathrm{br} \mathrm{s}, 2 \mathrm{H}) ; 2.39(\mathrm{~s}, 3 \mathrm{H}) ;{ }^{13} \mathrm{C} \mathrm{NMR}$ 
$\left(\mathrm{CDCl}_{3}\right) \delta$ 147.4, 128.6, 137.2, 130.4, 129.6, 128.3, 127.9, 124.8, 117.2, 116.6, 109.7, 21.6; IR (KBr) 3282, 3082, 1651, $1527 \mathrm{~cm}^{-1}$; MS (APCI) $\mathrm{m} / \mathrm{z}(\%) 224.1\left(\mathrm{M}^{+}+\mathrm{H}, 100\right), 208.1\left(\mathrm{M}^{+}-\mathrm{CH}_{3}\right.$, 40), $207.1\left(\mathrm{M}^{+}-\mathrm{NH}_{2}, 40\right)$.

3-Amino-2-phenyl-2H -indazole (7c): Diazene $5 \mathbf{c}(75 \mathrm{mg}, 0.362 \mathrm{mmol})$ and $\mathrm{SnCl}_{2} \cdot \mathrm{H}_{2} \mathrm{O}(0.343$ $\mathrm{g}, 1.81 \mathrm{mmol})$ in EtOH were reacted according to general procedure C. Upon concentration, $7 \mathrm{c}^{3,4}$ (67 mg, 95\%) was obtained as a tan solid: $\mathrm{mp} 142.5-143.3{ }^{\circ} \mathrm{C} ;{ }^{1} \mathrm{H} \mathrm{NMR}\left(\mathrm{CDCl}_{3}\right) \delta$ 7.67-7.65 (m, 2H), 7.60-7.42 (m, 5H), $7.26(\mathrm{td}, J=8.7,2.4 \mathrm{~Hz}, 1 \mathrm{H}) 6.89(\mathrm{td}, J=8.7,2.4 \mathrm{~Hz}, 1 \mathrm{H}) 4.36(\mathrm{br} \mathrm{s}$, $2 \mathrm{H}) ;{ }^{13} \mathrm{C} \mathrm{NMR}\left(\mathrm{CDCl}_{3}\right) \delta 148.4,138.4,138.1,129.6,128.4,127.5,124.8,119.3,118.5,116.7$, 109.5; IR (KBr) 3280, 2920, 1624, $1515 \mathrm{~cm}^{-1}$; MS (APCI) $\mathrm{m} / z$ (\%) $210.1\left(\mathrm{M}^{+}+\mathrm{H}, 100\right), 193.1$ $\left(\mathrm{M}^{+}-\mathrm{NH}_{2}, 60\right)$.

3-Amino-5-chloro-2-phenyl-2H-indazole (7d): Diazene 5d (75 mg, $0.310 \mathrm{mmol})$ and $\mathrm{SnCl}_{2} \cdot \mathrm{H}_{2} \mathrm{O}(0.294 \mathrm{~g}, 1.55 \mathrm{mmol})$ in $\mathrm{EtOH}(30 \mathrm{~mL})$ were reacted according to general procedure C. Upon concentration the crude product was chromatographed on silica ( $5 \% \mathrm{EtOAc} / \mathrm{CH}_{2} \mathrm{Cl}_{2}$ ) to afford 7d (72 mg, 94\%) as a light tan solid: mp 182.5-183.5 ${ }^{\circ} \mathrm{C} ;{ }^{1} \mathrm{H}$ NMR $\left(\mathrm{CDCl}_{3}\right) \delta 7.65$ (dt, $J=$ 7.8, $1.5 \mathrm{~Hz}, 2 \mathrm{H}), 7.59-7.52$ (m. 2H), 7.50-7.44 (m, 3H), 7.16 (dd, $J=9.5,1.8 \mathrm{~Hz}, 1 \mathrm{H}$ ), 4.30 (br s, $2 \mathrm{H}) ;{ }^{13} \mathrm{C} \mathrm{NMR}\left(\mathrm{CDCl}_{3}\right) \delta 146.7,138.1,137.8,129.7,128.8,124.9,123.9,118.4,118.0,109.8$, 104.1; IR (KBr) 3357, 3150, 1622, $1509 \mathrm{~cm}^{-1}$; MS (APCI) $\mathrm{m} / z$ (\%) $244.0\left(\mathrm{M}^{+}+\mathrm{H}, 100\right), 227.0$ $\left(\mathrm{M}^{+}-\mathrm{NH}_{2}, 20\right), 208.1\left(\mathrm{M}^{+}-\mathrm{Cl}, 60\right), 192.0\left(\mathrm{M}^{+}-\mathrm{NH}_{2} \mathrm{Cl}, 80\right)$.

3-Amino-5-methoxy-2-phenyl-2H-indazole (7e): Diazene 5 e $(75 \mathrm{mg}, 0.0 .316 \mathrm{mmol})$ and $\mathrm{SnCl}_{2} \cdot \mathrm{H}_{2} \mathrm{O}(0.300 \mathrm{~g}, 1.58 \mathrm{mmol})$ in $\mathrm{EtOH}(30 \mathrm{~mL})$ were reacted according to general procedure C. Upon concentration the crude material was chromatographed on silica (10\% EtOAc/ $\mathrm{CH}_{2} \mathrm{Cl}_{2}$ ) to afford $7 \mathrm{e}(56 \mathrm{mg}, 74 \%)$ as a tan solid: $\mathrm{mp} 168.5-169.7{ }^{\circ} \mathrm{C} ;{ }^{1} \mathrm{H} \mathrm{NMR}\left(\mathrm{CDCl}_{3}\right) \delta 7.67(\mathrm{~d}, J=8.0$ $\mathrm{Hz}, 2 \mathrm{H}), 7.53(\mathrm{t}, J=8.0 \mathrm{~Hz}, 2 \mathrm{H}), 7.47-7.41(\mathrm{~m}, 2 \mathrm{H}), 6.99(\mathrm{dd}, J=9.5,2.5 \mathrm{~Hz}, 1 \mathrm{H}), 6.66(\mathrm{~d}, J=$ $2.5 \mathrm{~Hz}, 1 \mathrm{H}), 4.11$ (br s, 2H), 3.82 (s, 3H); ${ }^{13} \mathrm{C} \mathrm{NMR}\left(\mathrm{CDCl}_{3}\right) \delta$ 153.1, 145.5, 138.7, 137.0129 .5 , 128.2, 124.8, 122.3, 118.6, 109.0, 95.6, 55.4; IR (KBr) 3356, 3159, 1641, 1519, $1219 \mathrm{~cm}^{-1}$; MS (APCI) $\mathrm{m} / z(\%) 240.1\left(\mathrm{M}^{+}+\mathrm{H}, 60\right), 223.0\left(\mathrm{M}^{+}-\mathrm{NH}_{2}, 20\right), 208.1\left(\mathrm{M}^{+}-\mathrm{CH}_{3} \mathrm{O}, 30\right)$.

3-Amino-2-(4-bromophenyl)-5-methoxy-2H-indazole (7f): Diazene $5 f(75 \mathrm{mg}, 0.237 \mathrm{mmol}$ ) and $\mathrm{SnCl}_{2} \bullet \mathrm{H}_{2} \mathrm{O}(0.225 \mathrm{~g}, 1.18 \mathrm{mmol})$ in $\mathrm{EtOH}(30 \mathrm{~mL})$ were reacted according to general procedure C. Upon concentration the crude product was chromatographed on silica (10-15\% EtOAc/ $\mathrm{CH}_{2} \mathrm{Cl}_{2}$ ) to afford $7 \mathbf{f}(58 \mathrm{mg}, 76 \%)$ as a light tan solid: mp 199.4-200.1 ${ }^{\circ} \mathrm{C} ;{ }^{1} \mathrm{H}$ NMR $\left(\right.$ THF-d $\left._{8}\right) \delta 7.79(\mathrm{~d}, J=8.7 \mathrm{~Hz}, 2 \mathrm{H}), 7.64(\mathrm{~d}, J=8.7 \mathrm{~Hz}, 2 \mathrm{H}), 7.23(\mathrm{~d}, J=9.0 \mathrm{~Hz}, 1 \mathrm{H}), 6.82-6.80$ 
(m, 2H), 5.24 (br s, 2H), 3.76 (s, 3H); ${ }^{13} \mathrm{C}$ NMR $\left(\mathrm{THF}_{-} \mathrm{d}_{8}\right) \delta$ 153.6, 146.8, 140.6, 149.9, 132.9, 126.4, 122.8, 120.8, 119.0, 110.1, 97.0, 55.4; IR (KBr) 3353, 3172, 1644, 1518, $1211 \mathrm{~cm}^{-1}$; MS (APCI) $m / z(\%) 318.0\left(\mathrm{M}^{+}+\mathrm{H}, 100\right), 239.1\left(\mathrm{M}^{+}+\mathrm{H}-\mathrm{Br}, 100\right)$.

3-Amino-2-(4-fluorophenyl)-5-methoxy-2H-indazole (7g): Diazene 5g (75 mg, $0.294 \mathrm{mmol}$ ) and $\mathrm{SnCl}_{2} \cdot \mathrm{H}_{2} \mathrm{O}(0.280 \mathrm{~g}, 1.47 \mathrm{mmol})$ in $\mathrm{EtOH}(30 \mathrm{~mL})$ were reacted according to general procedure C. Upon concentration the crude product was chromatographed on silica (10\% EtOAc/ $\left.\mathrm{CH}_{2} \mathrm{Cl}_{2}\right)$ to afford $7 \mathbf{g}(60 \mathrm{mg}, 79 \%)$ as a light tan solid: $\mathrm{mp} 204.1-205.3{ }^{\circ} \mathrm{C} ;{ }^{1} \mathrm{H}$ NMR $\left(\mathrm{CDCl}_{3}\right) \delta 7.68-7.63(\mathrm{~m}, 2 \mathrm{H}), 7.43(\mathrm{~d}, J=9.3 \mathrm{~Hz}, 1 \mathrm{H}), 7.25-7.19(\mathrm{~m}, 2 \mathrm{H}), 7.00(\mathrm{dd}, J=9.3,2.4$ $\mathrm{Hz}, 1 \mathrm{H}), 6.67(\mathrm{~d}, J=2.4 \mathrm{~Hz}, 1 \mathrm{H}), 4.08(\mathrm{br} \mathrm{s}, 2 \mathrm{H}), 3.83(\mathrm{~s}, 3 \mathrm{H}) ;{ }^{13} \mathrm{C} \mathrm{NMR}\left(\mathrm{CDCl}_{3}\right) \delta 161.1,154.3$ $(\mathrm{d}, J=1330.0 \mathrm{~Hz}) 153.3,135.9(\mathrm{~d}, J=181.0 \mathrm{~Hz}), 134.7,126.9,126.7,119.5(\mathrm{~d}, J=463.3 \mathrm{~Hz})$, $117.5(\mathrm{~d}, J=141.2 \mathrm{~Hz}), 116.4,109.2,95.6,55.4$; IR (KBr) 3365, 3137, 1654, $1519 \mathrm{~cm}^{-1}$; MS (APCI) $m / z(\%) 258.0\left(\mathrm{M}^{+}+\mathrm{H}, 90\right), 241.8\left(\mathrm{M}^{+}-\mathrm{NH}_{2}, 20\right), 210.0\left(\mathrm{M}^{+}-\mathrm{CH}_{5} \mathrm{NO}, 25\right)$.

3-Amino-2-(4-carbomethoxyphenyl)-5-methoxy-2H-indazole (7h): Diazene 5 h (75 mg, 0.254 $\mathrm{mmol})$ and $\mathrm{SnCl}_{2} \bullet \mathrm{H}_{2} \mathrm{O}(0.241 \mathrm{~g}, 1.27 \mathrm{mmol})$ in $\mathrm{EtOH}(30 \mathrm{~mL})$ were reacted according to general procedure C. Upon concentration the crude product was chromatographed on silica (35\% EtOAc/ $\left.\mathrm{CH}_{2} \mathrm{Cl}_{2}\right)$ to afford $7 \mathbf{h}(64 \mathrm{mg}, 84 \%)$ as a light tan solid: $\mathrm{mp} 228.2-229.4{ }^{\circ} \mathrm{C} ;{ }^{1} \mathrm{H} \mathrm{NMR}$ $\left(\mathrm{THF}-\mathrm{d}_{8}\right) \delta 8.12(\mathrm{~d}, J=8.2 \mathrm{~Hz}, 2 \mathrm{H}), 8.01(\mathrm{~d}, J=8.2 \mathrm{~Hz}, 2 \mathrm{H}), 7.23(\mathrm{~d}, J=9.5 \mathrm{~Hz}, 1 \mathrm{H}), 6.83-6.80$ (m, 2H), 5.34 (br s, 2H), 3.89 (s, 3H), 3.76 (s, 3H); ${ }^{13} \mathrm{C}$ NMR $\left(\mathrm{THF}_{\mathrm{d}}\right) \delta$ 166.5, 153.7, 147.1, 145.2, 140.2, 131.1, 129.0, 123.80, 123.78, 123.2, 119.1, 110.3, 96.9, 55.4, 52.2; IR (KBr) 3353, 3167, 1714, 1641, $1521 \mathrm{~cm}^{-1}$; MS (APCI) $\mathrm{m} / z(\%) 298.1\left(\mathrm{M}^{+}+\mathrm{H}, 60\right), 266.1\left(\mathrm{M}^{+}-\mathrm{CH}_{3} \mathrm{O}, 30\right)$. 3-Amino-2-(4-cyanophenyl)-5-methoxy-2H-indazole (7i): Diazene 5i (75 mg, $0.310 \mathrm{mmol}$ ) and $\mathrm{SnCl}_{2} \cdot \mathrm{H}_{2} \mathrm{O}(0.294 \mathrm{~g}, 1.55 \mathrm{mmol})$ in $\mathrm{EtOH}(30 \mathrm{~mL})$ were reacted according to general procedure C. Upon concentration the crude product was chromatographed on silica (5\% EtOAc/ $\mathrm{CH}_{2} \mathrm{Cl}_{2}$ ) to afford $\mathbf{5 i}$ (72 mg, 94\%) as a light tan solid: mp 192.3-193.9 ${ }^{\circ} \mathrm{C} ;{ }^{1} \mathrm{H}$ NMR $\left(\mathrm{CDCl}_{3}\right) \delta 7.97(\mathrm{dt}, J=9.0,1.5 \mathrm{~Hz}, 2 \mathrm{H}), 7.82(\mathrm{dt}, J=9.0,1.5 \mathrm{~Hz}, 2 \mathrm{H}), 7.44(\mathrm{~d}, J=9.3 \mathrm{~Hz}, 1 \mathrm{H})$, $7.00(\mathrm{dd}, J=9.3,2.4 \mathrm{~Hz}, 1 \mathrm{H}), 6.62(\mathrm{~d}, J=2.4 \mathrm{~Hz}, 1 \mathrm{H}), 4.11$ (br s, $2 \mathrm{H}), 3.84(\mathrm{~s}, 3 \mathrm{H}) ;{ }^{13} \mathrm{C} \mathrm{NMR}$ $\left(\mathrm{CDCl}_{3}\right) \delta 154.3,147.8,144.2,142.5,134.8,126.1,125.0,119.4,118.0,112.4,110.2,110.2$, 98.2, 56.0; IR (KBr) 3371, 3169, 2225, 1606, $1521 \mathrm{~cm}^{-1}$; MS (APCI) $m / z(\%) 265.1\left(\mathrm{M}^{+}+\mathrm{H}\right.$, 100), $248.1\left(\mathrm{M}^{+}-\mathrm{NH}_{2}, 20\right), 222.1\left(\mathrm{M}^{+}-\mathrm{CH}_{2} \mathrm{~N}_{2}, 100\right)$.

3-Amino-5-methoxy-2-(4-nitrophenyl)-2H-indazole (7j): Diazene 5j (75 mg, $0.266 \mathrm{mmol}$ ) and $\mathrm{SnCl}_{2} \bullet \mathrm{H}_{2} \mathrm{O}(0.252 \mathrm{~g}, 1.33 \mathrm{mmol})$ in $\mathrm{EtOH}(30 \mathrm{~mL})$ were reacted according to general procedure 
C. Upon concentration the crude product was chromatographed on silica $\left(3 \% \mathrm{CH}_{2} \mathrm{Cl}_{2} / \mathrm{EtOAc}\right)$ to afford $7 \mathbf{j}$ (61 mg, 80\%) as a light tan solid: mp 216.1-217.3 ${ }^{\circ} \mathrm{C} ;{ }^{1} \mathrm{H}$ NMR $\left(\right.$ THF- $\left.\mathrm{d}_{8}\right) \delta 7.35$ (d, $J=$ $8.5 \mathrm{~Hz}, 2 \mathrm{H}), 7.19(\mathrm{~d}, J=8.5 \mathrm{~Hz}, 2 \mathrm{H}), 6.79(\mathrm{~d}, J=2.0 \mathrm{~Hz}, 1 \mathrm{H}), 6.75(\mathrm{dd}, J=8.7,2.0 \mathrm{~Hz}, 1 \mathrm{H})$, $6.65(\mathrm{~d}, J=8.7 \mathrm{~Hz}, 1 \mathrm{H}), 4.93(\mathrm{br} \mathrm{s}, 2 \mathrm{H}), 3.73(\mathrm{~s}, 3 \mathrm{H}),{ }^{13} \mathrm{C}$ NMR $\left(\mathrm{THF}_{8} \mathrm{~d}_{8}\right) \delta$ 153.1, 149.0, 145.9, 139.2, 130.3, 126.3, 121.3, 118.8, 114.8, 103.2, 97.2, 55.4; IR(KBr) 3400, 2961, 1644, $1508 \mathrm{~cm}^{-}$ ${ }^{1}$; MS (APCI) $m / z(\%) 285.1\left(\mathrm{M}^{+}+\mathrm{H}, 30\right), 238.0\left(\mathrm{M}^{+}-\mathrm{NO}_{2}, 50\right)$.

\section{References}

(1) Shirtcliff, L. D.; Weakley, T. J. R.; Haley, M. M.; Kohler, F.; Herges, R. J. Org. Chem 2004, 69, 6979-6985.

(2) Roling, P. J. Org. Chem. 1975, 40, 2421-2425.

(3) Partridge, M. W.; Stevens, M. F. G. J. Chem. Soc. 1964, 3663-3669.

(4) Alberti, A.; Bedogni, N.; Benaglia, M.; Leardini, R.; Nanni, D.; Pedulli, G. F.; Tundo, A.; Zanardi, G. J. Org. Chem. 1992, 57, 607-613. 
Molecular structure of imine $\mathbf{6 j}$; ellipsoids drawn at the $30 \%$ probability level.

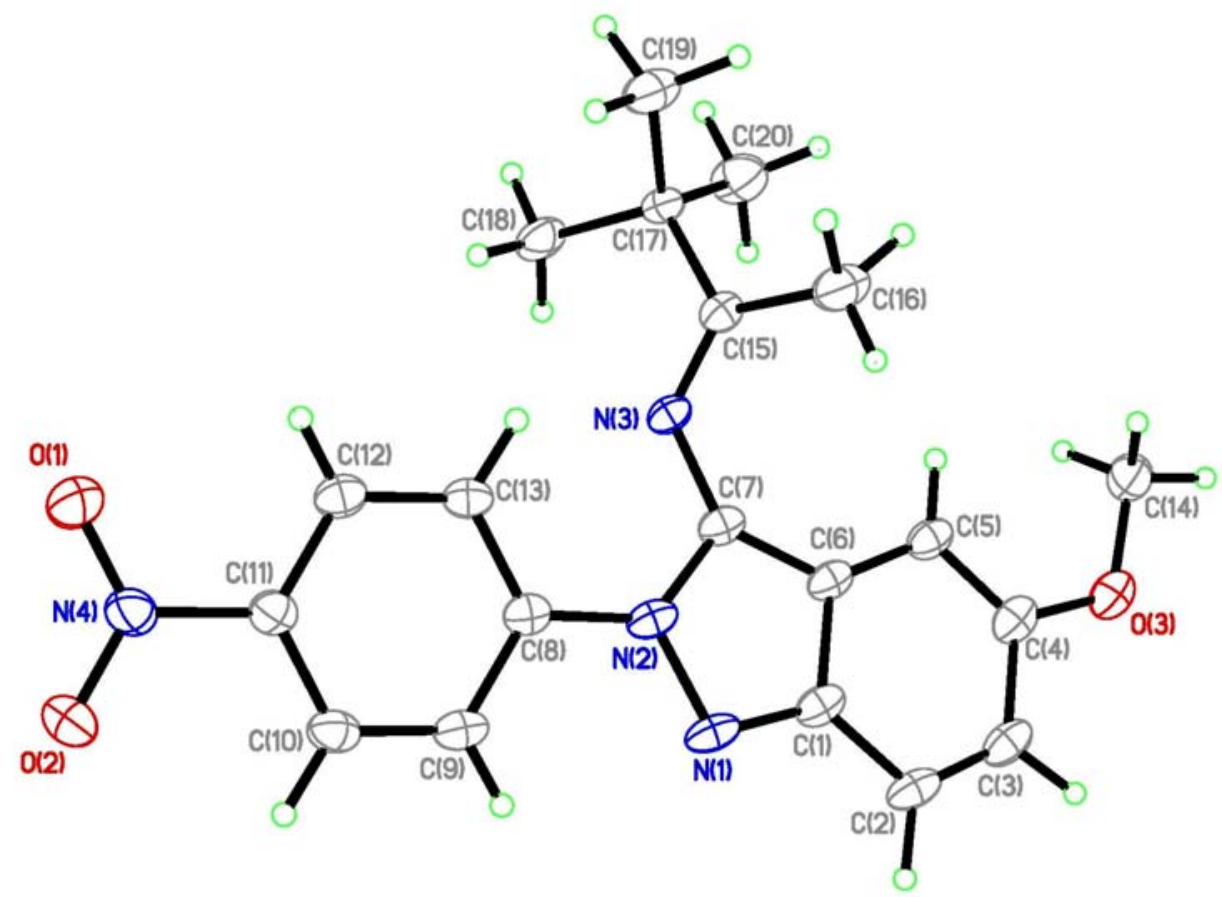


Table 1. Crystal data and structure refinement for $\mathbf{6 j}$.

Identification code

Empirical formula

Formula weight

Temperature

Wavelength

Crystal system

Space group

Unit cell dimensions

Volume

Z

Density (calculated)

Absorption coefficient

$\mathrm{F}(000)$

Crystal size

Theta range for data collection

Index ranges

Reflections collected

Independent reflections

Completeness to theta $=25.00 \infty$

Absorption correction

Max. and min. transmission

Refinement method

Data / restraints / parameters

Goodness-of-fit on $\mathrm{F}^{2}$

Final $\mathrm{R}$ indices $[\mathrm{I}>2 \operatorname{sigma}(\mathrm{I})]$

$\mathrm{R}$ indices (all data)

Largest diff. peak and hole lds 7

C20 H22 N4 O3

366.42

153(2) K

$0.71073 \AA$

Triclinic

P-1

$\mathrm{a}=9.8124(13) \AA$

$\alpha=113.836(3)^{\circ}$

$\mathrm{b}=10.8017(14) \AA$

$\beta=115.303(3)^{\circ}$

$\mathrm{c}=11.0897(13) \AA$

$\gamma=91.970(3)^{\circ}$

$941.0(2) \AA^{3}$

2

$1.293 \mathrm{Mg} / \mathrm{m}^{3}$

$0.089 \mathrm{~mm}^{-1}$

388

$0.29 \times 0.18 \times 0.02 \mathrm{~mm}^{3}$

2.13 to $25.00^{\circ}$

$-11<=\mathrm{h}<=11,-12<=\mathrm{k}<=12,-13<=1<=13$

7416

$3297[\mathrm{R}(\mathrm{int})=0.0291]$

$99.5 \%$

Semi-empirical from equivalents

1.000 and 0.870

Full-matrix least-squares on $\mathrm{F}^{2}$

3297 / 0 / 332

1.021

$\mathrm{R} 1=0.0483, \mathrm{wR} 2=0.1024$

$\mathrm{R} 1=0.0838, \mathrm{wR} 2=0.1209$

0.209 and $-0.156 \mathrm{e}^{3}$ 
Table 2. Atomic coordinates ( $\left.\times 10^{4}\right)$ and equivalent isotropic displacement parameters $\left(\AA^{2} \times 10^{3}\right)$ for $\mathbf{6 j}$. $U(e q)$ is defined as one third of the trace of the orthogonalized $U^{i j}$ tensor.

\begin{tabular}{|c|c|c|c|c|}
\hline & $\mathrm{x}$ & $\mathrm{y}$ & z & $\mathrm{U}(\mathrm{eq})$ \\
\hline $\mathrm{O}(1)$ & $141(2)$ & $12214(2)$ & $6280(2)$ & $63(1)$ \\
\hline $\mathrm{O}(2)$ & $152(3)$ & 14081(2) & $6032(2)$ & $82(1)$ \\
\hline $\mathrm{O}(3)$ & $4067(2)$ & $7836(2)$ & $-3327(2)$ & $52(1)$ \\
\hline $\mathrm{N}(1)$ & $1925(2)$ & $11204(2)$ & $333(2)$ & $41(1)$ \\
\hline $\mathrm{N}(2)$ & 2090(2) & $10536(2)$ & $1195(2)$ & $37(1)$ \\
\hline $\mathrm{N}(3)$ & $3170(2)$ & $8709(2)$ & $1689(2)$ & $33(1)$ \\
\hline $\mathrm{N}(4)$ & $350(2)$ & $12904(2)$ & $5703(2)$ & $48(1)$ \\
\hline $\mathrm{C}(1)$ & $2415(2)$ & $10427(2)$ & $-629(3)$ & $38(1)$ \\
\hline$C(2)$ & $2547(3)$ & 10689(3) & $-1738(3)$ & $44(1)$ \\
\hline$C(3)$ & 3111(3) & $9795(3)$ & $-2567(3)$ & $45(1)$ \\
\hline$C(4)$ & $3549(2)$ & $8624(3)$ & $-2364(3)$ & $42(1)$ \\
\hline$C(5)$ & $3448(2)$ & $8352(3)$ & $-1298(2)$ & $39(1)$ \\
\hline$C(6)$ & $2856(2)$ & $9268(2)$ & $-420(2)$ & $34(1)$ \\
\hline$C(7)$ & $2645(2)$ & $9364(2)$ & $777(2)$ & $34(1)$ \\
\hline$C(8)$ & $1685(2)$ & $11118(2)$ & $2354(2)$ & $33(1)$ \\
\hline$C(9)$ & 1497(3) & $12477(3)$ & 2794(3) & $41(1)$ \\
\hline$C(10)$ & 1062(3) & 13061(3) & $3888(3)$ & $43(1)$ \\
\hline$C(11)$ & $841(2)$ & 12293(2) & $4559(2)$ & $38(1)$ \\
\hline$C(12)$ & 1041(3) & $10962(3)$ & $4152(3)$ & $40(1)$ \\
\hline$C(13)$ & 1449(3) & $10360(3)$ & $3039(3)$ & $37(1)$ \\
\hline$C(14)$ & 4411(4) & $6583(3)$ & $-3277(3)$ & $54(1)$ \\
\hline$C(15)$ & $2869(2)$ & 7393(2) & $1179(2)$ & $34(1)$ \\
\hline$C(16)$ & $1750(4)$ & $6346(4)$ & $-422(3)$ & $67(1)$ \\
\hline$C(17)$ & $3708(2)$ & $6813(2)$ & $2270(2)$ & $35(1)$ \\
\hline$C(18)$ & 4567(4) & 7985(3) & $3886(3)$ & $54(1)$ \\
\hline$C(19)$ & 2579(4) & $5721(3)$ & 2165(4) & $57(1)$ \\
\hline $\mathrm{C}(20)$ & 4920(4) & $6163(4)$ & 1870(4) & $65(1)$ \\
\hline
\end{tabular}


Table 3. Bond lengths $[\AA]$ and angles $\left[{ }^{\circ}\right]$ for $\mathbf{6} \mathbf{j}$.

\begin{tabular}{|c|c|}
\hline $\mathrm{O}(1)-\mathrm{N}(4)$ & $1.222(2)$ \\
\hline $\mathrm{O}(2)-\mathrm{N}(4)$ & $1.221(2)$ \\
\hline $\mathrm{O}(3)-\mathrm{C}(4)$ & $1.372(3)$ \\
\hline $\mathrm{O}(3)-\mathrm{C}(14)$ & $1.422(3)$ \\
\hline $\mathrm{N}(1)-\mathrm{C}(1)$ & $1.348(3)$ \\
\hline $\mathrm{N}(1)-\mathrm{N}(2)$ & $1.375(2)$ \\
\hline $\mathrm{N}(2)-\mathrm{C}(7)$ & $1.380(3)$ \\
\hline $\mathrm{N}(2)-\mathrm{C}(8)$ & $1.416(3)$ \\
\hline $\mathrm{N}(3)-\mathrm{C}(15)$ & $1.267(3)$ \\
\hline $\mathrm{N}(3)-\mathrm{C}(7)$ & $1.395(3)$ \\
\hline $\mathrm{N}(4)-\mathrm{C}(11)$ & $1.464(3)$ \\
\hline$C(1)-C(6)$ & $1.411(3)$ \\
\hline $\mathrm{C}(1)-\mathrm{C}(2)$ & $1.423(3)$ \\
\hline$C(2)-C(3)$ & $1.356(3)$ \\
\hline $\mathrm{C}(2)-\mathrm{H}(2)$ & $0.99(2)$ \\
\hline $\mathrm{C}(3)-\mathrm{C}(4)$ & $1.421(3)$ \\
\hline $\mathrm{C}(3)-\mathrm{H}(3)$ & $1.00(3)$ \\
\hline$C(4)-C(5)$ & $1.368(3)$ \\
\hline$C(5)-C(6)$ & $1.417(3)$ \\
\hline $\mathrm{C}(5)-\mathrm{H}(5)$ & $0.94(2)$ \\
\hline$C(6)-C(7)$ & $1.394(3)$ \\
\hline$C(8)-C(13)$ & $1.393(3)$ \\
\hline $\mathrm{C}(8)-\mathrm{C}(9)$ & $1.394(3)$ \\
\hline $\mathrm{C}(9)-\mathrm{C}(10)$ & $1.376(4)$ \\
\hline $\mathrm{C}(9)-\mathrm{H}(9)$ & $0.93(2)$ \\
\hline $\mathrm{C}(10)-\mathrm{C}(11)$ & $1.383(3)$ \\
\hline $\mathrm{C}(10)-\mathrm{H}(10)$ & $0.98(2)$ \\
\hline $\mathrm{C}(11)-\mathrm{C}(12)$ & $1.371(3)$ \\
\hline$C(12)-C(13)$ & $1.378(3)$ \\
\hline $\mathrm{C}(12)-\mathrm{H}(12)$ & $0.90(2)$ \\
\hline $\mathrm{C}(13)-\mathrm{H}(13)$ & $0.96(2)$ \\
\hline C(14)-H(14A) & $1.03(3)$ \\
\hline C(14)-H(14B) & $1.05(3)$ \\
\hline $\mathrm{C}(14)-\mathrm{H}(14 \mathrm{C})$ & $1.01(3)$ \\
\hline
\end{tabular}




\begin{tabular}{|c|c|}
\hline$C(15)-C(16)$ & $1.504(4)$ \\
\hline$C(15)-C(17)$ & $1.526(3)$ \\
\hline $\mathrm{C}(16)-\mathrm{H}(16 \mathrm{~A})$ & $1.03(5)$ \\
\hline $\mathrm{C}(16)-\mathrm{H}(16 \mathrm{~B})$ & $0.92(3)$ \\
\hline $\mathrm{C}(16)-\mathrm{H}(16 \mathrm{C})$ & $1.00(3)$ \\
\hline $\mathrm{C}(17)-\mathrm{C}(19)$ & $1.524(3)$ \\
\hline $\mathrm{C}(17)-\mathrm{C}(18)$ & $1.526(3)$ \\
\hline$C(17)-C(20)$ & $1.536(3)$ \\
\hline $\mathrm{C}(18)-\mathrm{H}(18 \mathrm{~A})$ & $1.10(3)$ \\
\hline $\mathrm{C}(18)-\mathrm{H}(18 \mathrm{~B})$ & $1.01(3)$ \\
\hline $\mathrm{C}(18)-\mathrm{H}(18 \mathrm{C})$ & $0.99(3)$ \\
\hline $\mathrm{C}(19)-\mathrm{H}(19 \mathrm{~A})$ & $1.00(3)$ \\
\hline C(19)-H(19B) & $1.01(3)$ \\
\hline $\mathrm{C}(19)-\mathrm{H}(19 \mathrm{C})$ & $1.03(3)$ \\
\hline $\mathrm{C}(20)-\mathrm{H}(20 \mathrm{~A})$ & $1.06(3)$ \\
\hline $\mathrm{C}(20)-\mathrm{H}(20 \mathrm{~B})$ & $1.04(3)$ \\
\hline $\mathrm{C}(20)-\mathrm{H}(20 \mathrm{C})$ & $1.02(3)$ \\
\hline $\mathrm{C}(4)-\mathrm{O}(3)-\mathrm{C}(14)$ & $116.59(19)$ \\
\hline $\mathrm{C}(1)-\mathrm{N}(1)-\mathrm{N}(2)$ & $103.37(17)$ \\
\hline $\mathrm{N}(1)-\mathrm{N}(2)-\mathrm{C}(7)$ & $113.05(18)$ \\
\hline $\mathrm{N}(1)-\mathrm{N}(2)-\mathrm{C}(8)$ & $117.63(18)$ \\
\hline $\mathrm{C}(7)-\mathrm{N}(2)-\mathrm{C}(8)$ & $129.32(17)$ \\
\hline $\mathrm{C}(15)-\mathrm{N}(3)-\mathrm{C}(7)$ & $123.32(19)$ \\
\hline $\mathrm{O}(2)-\mathrm{N}(4)-\mathrm{O}(1)$ & $122.6(2)$ \\
\hline $\mathrm{O}(2)-\mathrm{N}(4)-\mathrm{C}(11)$ & $118.4(2)$ \\
\hline $\mathrm{O}(1)-\mathrm{N}(4)-\mathrm{C}(11)$ & $118.9(2)$ \\
\hline $\mathrm{N}(1)-\mathrm{C}(1)-\mathrm{C}(6)$ & $112.72(19)$ \\
\hline $\mathrm{N}(1)-\mathrm{C}(1)-\mathrm{C}(2)$ & $126.9(2)$ \\
\hline$C(6)-C(1)-C(2)$ & $120.4(2)$ \\
\hline$C(3)-C(2)-C(1)$ & $117.6(2)$ \\
\hline $\mathrm{C}(3)-\mathrm{C}(2)-\mathrm{H}(2)$ & $122.9(13)$ \\
\hline $\mathrm{C}(1)-\mathrm{C}(2)-\mathrm{H}(2)$ & $119.4(13)$ \\
\hline$C(2)-C(3)-C(4)$ & $122.1(2)$ \\
\hline $\mathrm{C}(2)-\mathrm{C}(3)-\mathrm{H}(3)$ & $122.9(14)$ \\
\hline $\mathrm{C}(4)-\mathrm{C}(3)-\mathrm{H}(3)$ & $114.9(14)$ \\
\hline
\end{tabular}




\begin{tabular}{|c|c|}
\hline $\mathrm{C}(5)-\mathrm{C}(4)-\mathrm{O}(3)$ & $125.2(2)$ \\
\hline$C(5)-C(4)-C(3)$ & $121.5(2)$ \\
\hline $\mathrm{O}(3)-\mathrm{C}(4)-\mathrm{C}(3)$ & $113.2(2)$ \\
\hline$C(4)-C(5)-C(6)$ & $117.4(2)$ \\
\hline $\mathrm{C}(4)-\mathrm{C}(5)-\mathrm{H}(5)$ & $121.7(12)$ \\
\hline $\mathrm{C}(6)-\mathrm{C}(5)-\mathrm{H}(5)$ & $120.9(12)$ \\
\hline$C(7)-C(6)-C(1)$ & $105.14(19)$ \\
\hline$C(7)-C(6)-C(5)$ & $133.9(2)$ \\
\hline$C(1)-C(6)-C(5)$ & $120.9(2)$ \\
\hline $\mathrm{N}(2)-\mathrm{C}(7)-\mathrm{N}(3)$ & $121.17(19)$ \\
\hline $\mathrm{N}(2)-\mathrm{C}(7)-\mathrm{C}(6)$ & $105.71(17)$ \\
\hline $\mathrm{N}(3)-\mathrm{C}(7)-\mathrm{C}(6)$ & $131.6(2)$ \\
\hline $\mathrm{C}(13)-\mathrm{C}(8)-\mathrm{C}(9)$ & $119.7(2)$ \\
\hline $\mathrm{C}(13)-\mathrm{C}(8)-\mathrm{N}(2)$ & $121.7(2)$ \\
\hline $\mathrm{C}(9)-\mathrm{C}(8)-\mathrm{N}(2)$ & $118.6(2)$ \\
\hline$C(10)-C(9)-C(8)$ & $120.1(2)$ \\
\hline $\mathrm{C}(10)-\mathrm{C}(9)-\mathrm{H}(9)$ & $121.3(14)$ \\
\hline $\mathrm{C}(8)-\mathrm{C}(9)-\mathrm{H}(9)$ & $118.6(14)$ \\
\hline $\mathrm{C}(9)-\mathrm{C}(10)-\mathrm{C}(11)$ & $119.3(2)$ \\
\hline $\mathrm{C}(9)-\mathrm{C}(10)-\mathrm{H}(10)$ & $121.2(15)$ \\
\hline $\mathrm{C}(11)-\mathrm{C}(10)-\mathrm{H}(10)$ & $119.5(15)$ \\
\hline$C(12)-C(11)-C(10)$ & $121.3(2)$ \\
\hline $\mathrm{C}(12)-\mathrm{C}(11)-\mathrm{N}(4)$ & $119.4(2)$ \\
\hline $\mathrm{C}(10)-\mathrm{C}(11)-\mathrm{N}(4)$ & $119.3(2)$ \\
\hline $\mathrm{C}(11)-\mathrm{C}(12)-\mathrm{C}(13)$ & $119.9(2)$ \\
\hline $\mathrm{C}(11)-\mathrm{C}(12)-\mathrm{H}(12)$ & $119.9(15)$ \\
\hline $\mathrm{C}(13)-\mathrm{C}(12)-\mathrm{H}(12)$ & $120.1(15)$ \\
\hline $\mathrm{C}(12)-\mathrm{C}(13)-\mathrm{C}(8)$ & $119.8(2)$ \\
\hline $\mathrm{C}(12)-\mathrm{C}(13)-\mathrm{H}(13)$ & $120.7(12)$ \\
\hline $\mathrm{C}(8)-\mathrm{C}(13)-\mathrm{H}(13)$ & $119.5(12)$ \\
\hline $\mathrm{O}(3)-\mathrm{C}(14)-\mathrm{H}(14 \mathrm{~A})$ & 109.3(13) \\
\hline $\mathrm{O}(3)-\mathrm{C}(14)-\mathrm{H}(14 \mathrm{~B})$ & $104.7(14)$ \\
\hline $\mathrm{H}(14 \mathrm{~A})-\mathrm{C}(14)-\mathrm{H}(14 \mathrm{~B})$ & 109.6(19) \\
\hline $\mathrm{O}(3)-\mathrm{C}(14)-\mathrm{H}(14 \mathrm{C})$ & $113.0(15)$ \\
\hline $\mathrm{H}(14 \mathrm{~A})-\mathrm{C}(14)-\mathrm{H}(14 \mathrm{C})$ & $109(2)$ \\
\hline $\mathrm{H}(14 \mathrm{~B})-\mathrm{C}(14)-\mathrm{H}(14 \mathrm{C})$ & $111(2)$ \\
\hline
\end{tabular}




\begin{tabular}{|c|c|}
\hline$N(3)-C(15)-C(16)$ & $125.4(2)$ \\
\hline $\mathrm{N}(3)-\mathrm{C}(15)-\mathrm{C}(17)$ & $117.73(19)$ \\
\hline $\mathrm{C}(16)-\mathrm{C}(15)-\mathrm{C}(17)$ & $116.9(2)$ \\
\hline $\mathrm{C}(15)-\mathrm{C}(16)-\mathrm{H}(16 \mathrm{~A})$ & $115(3)$ \\
\hline $\mathrm{C}(15)-\mathrm{C}(16)-\mathrm{H}(16 \mathrm{~B})$ & $111.5(16)$ \\
\hline $\mathrm{H}(16 \mathrm{~A})-\mathrm{C}(16)-\mathrm{H}(16 \mathrm{~B})$ & $114(3)$ \\
\hline $\mathrm{C}(15)-\mathrm{C}(16)-\mathrm{H}(16 \mathrm{C})$ & $107.8(18)$ \\
\hline $\mathrm{H}(16 \mathrm{~A})-\mathrm{C}(16)-\mathrm{H}(16 \mathrm{C})$ & $95(3)$ \\
\hline H(16B)-C(16)-H(16C) & $113(2)$ \\
\hline $\mathrm{C}(19)-\mathrm{C}(17)-\mathrm{C}(15)$ & $111.60(19)$ \\
\hline $\mathrm{C}(19)-\mathrm{C}(17)-\mathrm{C}(18)$ & $108.8(2)$ \\
\hline $\mathrm{C}(15)-\mathrm{C}(17)-\mathrm{C}(18)$ & $110.64(19)$ \\
\hline $\mathrm{C}(19)-\mathrm{C}(17)-\mathrm{C}(20)$ & $110.3(2)$ \\
\hline $\mathrm{C}(15)-\mathrm{C}(17)-\mathrm{C}(20)$ & $107.40(19)$ \\
\hline $\mathrm{C}(18)-\mathrm{C}(17)-\mathrm{C}(20)$ & $108.1(2)$ \\
\hline $\mathrm{C}(17)-\mathrm{C}(18)-\mathrm{H}(18 \mathrm{~A})$ & $108.3(17)$ \\
\hline $\mathrm{C}(17)-\mathrm{C}(18)-\mathrm{H}(18 \mathrm{~B})$ & $113.7(16)$ \\
\hline $\mathrm{H}(18 \mathrm{~A})-\mathrm{C}(18)-\mathrm{H}(18 \mathrm{~B})$ & $108(2)$ \\
\hline $\mathrm{C}(17)-\mathrm{C}(18)-\mathrm{H}(18 \mathrm{C})$ & $108.5(15)$ \\
\hline $\mathrm{H}(18 \mathrm{~A})-\mathrm{C}(18)-\mathrm{H}(18 \mathrm{C})$ & $110(2)$ \\
\hline H(18B)-C(18)-H(18C) & $108(2)$ \\
\hline $\mathrm{C}(17)-\mathrm{C}(19)-\mathrm{H}(19 \mathrm{~A})$ & $107.8(15)$ \\
\hline $\mathrm{C}(17)-\mathrm{C}(19)-\mathrm{H}(19 \mathrm{~B})$ & $110.2(15)$ \\
\hline H(19A)-C(19)-H(19B) & $104(2)$ \\
\hline C(17)-C(19)-H(19C) & $109.8(15)$ \\
\hline H(19A)-C(19)-H(19C) & $116(2)$ \\
\hline H(19B)-C(19)-H(19C) & $109(2)$ \\
\hline $\mathrm{C}(17)-\mathrm{C}(20)-\mathrm{H}(20 \mathrm{~A})$ & $111.3(17)$ \\
\hline $\mathrm{C}(17)-\mathrm{C}(20)-\mathrm{H}(20 \mathrm{~B})$ & $105.5(17)$ \\
\hline $\mathrm{H}(20 \mathrm{~A})-\mathrm{C}(20)-\mathrm{H}(20 \mathrm{~B})$ & $106(2)$ \\
\hline $\mathrm{C}(17)-\mathrm{C}(20)-\mathrm{H}(20 \mathrm{C})$ & $108.8(17)$ \\
\hline $\mathrm{H}(20 \mathrm{~A})-\mathrm{C}(20)-\mathrm{H}(20 \mathrm{C})$ & $112(2)$ \\
\hline $\mathrm{H}(20 \mathrm{~B})-\mathrm{C}(20)-\mathrm{H}(20 \mathrm{C})$ & $113(2)$ \\
\hline
\end{tabular}

Symmetry transformations used to generate equivalent atoms: 
Table 4. Anisotropic displacement parameters $\left(\AA^{2} \times 10^{3}\right)$ for $\mathbf{6 j}$. The anisotropic displacement factor exponent takes the form: $-2 \pi^{2}\left[h^{2} a^{* 2} U^{11}+\ldots+2 h k a^{*} b^{*} U^{12}\right]$

\begin{tabular}{|c|c|c|c|c|c|c|}
\hline & $\mathrm{U}^{11}$ & $\mathrm{U}^{22}$ & $\mathrm{U}^{33}$ & $U^{23}$ & $\mathrm{U}^{13}$ & $\mathrm{U}^{12}$ \\
\hline $\mathrm{O}(1)$ & $89(2)$ & $64(1)$ & $63(1)$ & $44(1)$ & $46(1)$ & $38(1)$ \\
\hline $\mathrm{O}(2)$ & $150(2)$ & $50(1)$ & $68(1)$ & $33(1)$ & $65(1)$ & $52(1)$ \\
\hline $\mathrm{O}(3)$ & $55(1)$ & $64(1)$ & $46(1)$ & $31(1)$ & $26(1)$ & $13(1)$ \\
\hline $\mathrm{N}(1)$ & $32(1)$ & $51(1)$ & $48(1)$ & $38(1)$ & $13(1)$ & $11(1)$ \\
\hline $\mathrm{N}(2)$ & $29(1)$ & $44(1)$ & $42(1)$ & $30(1)$ & $13(1)$ & $8(1)$ \\
\hline $\mathrm{N}(3)$ & $27(1)$ & $41(1)$ & $35(1)$ & $25(1)$ & $13(1)$ & $10(1)$ \\
\hline $\mathrm{N}(4)$ & $59(1)$ & $41(1)$ & $37(1)$ & $20(1)$ & $16(1)$ & $21(1)$ \\
\hline $\mathrm{C}(1)$ & $24(1)$ & $51(2)$ & $40(1)$ & $30(1)$ & $10(1)$ & $6(1)$ \\
\hline $\mathrm{C}(2)$ & $31(1)$ & $58(2)$ & $49(2)$ & $40(1)$ & $12(1)$ & $8(1)$ \\
\hline$C(3)$ & $31(1)$ & $64(2)$ & $43(2)$ & $35(1)$ & $12(1)$ & $5(1)$ \\
\hline $\mathrm{C}(4)$ & $27(1)$ & $57(2)$ & $39(1)$ & $26(1)$ & 11(1) & $4(1)$ \\
\hline$C(5)$ & $30(1)$ & $48(2)$ & $38(1)$ & $26(1)$ & $10(1)$ & $5(1)$ \\
\hline $\mathrm{C}(6)$ & $23(1)$ & $44(2)$ & $34(1)$ & $25(1)$ & $6(1)$ & $3(1)$ \\
\hline$C(7)$ & $24(1)$ & $41(1)$ & $36(1)$ & $24(1)$ & $9(1)$ & $6(1)$ \\
\hline $\mathrm{C}(8)$ & $22(1)$ & $38(1)$ & $35(1)$ & $20(1)$ & $6(1)$ & $5(1)$ \\
\hline $\mathrm{C}(9)$ & $37(1)$ & $41(2)$ & $48(2)$ & $29(1)$ & $14(1)$ & $7(1)$ \\
\hline $\mathrm{C}(10)$ & $43(1)$ & $36(2)$ & $42(1)$ & $20(1)$ & 11(1) & $10(1)$ \\
\hline $\mathrm{C}(11)$ & $34(1)$ & $37(1)$ & $33(1)$ & $17(1)$ & $8(1)$ & $10(1)$ \\
\hline $\mathrm{C}(12)$ & $35(1)$ & $44(2)$ & $43(1)$ & $27(1)$ & $15(1)$ & 11(1) \\
\hline$C(13)$ & $35(1)$ & $36(2)$ & $45(1)$ & $24(1)$ & $17(1)$ & $12(1)$ \\
\hline $\mathrm{C}(14)$ & $58(2)$ & $55(2)$ & $48(2)$ & $25(2)$ & $26(2)$ & $10(2)$ \\
\hline$C(15)$ & $27(1)$ & $40(2)$ & $35(1)$ & $20(1)$ & $13(1)$ & $4(1)$ \\
\hline$C(16)$ & $69(2)$ & $56(2)$ & $43(2)$ & $28(2)$ & $-2(2)$ & $-19(2)$ \\
\hline$C(17)$ & $39(1)$ & $37(1)$ & $41(1)$ & $25(1)$ & $21(1)$ & 13(1) \\
\hline $\mathrm{C}(18)$ & $59(2)$ & $57(2)$ & $39(2)$ & $31(2)$ & $10(1)$ & $6(2)$ \\
\hline$C(19)$ & $56(2)$ & $60(2)$ & $59(2)$ & $39(2)$ & $23(2)$ & $6(2)$ \\
\hline $\mathrm{C}(20)$ & $70(2)$ & $74(2)$ & $83(2)$ & $51(2)$ & $49(2)$ & $38(2)$ \\
\hline
\end{tabular}


Table 5. Hydrogen coordinates ( x $\left.10^{4}\right)$ and isotropic displacement parameters $\left(\AA^{2} \times 10^{3}\right)$ for $\mathbf{6 j}$.

\begin{tabular}{|c|c|c|c|c|}
\hline & $\mathrm{x}$ & $\mathrm{y}$ & z & $\mathrm{U}(\mathrm{eq})$ \\
\hline $\mathrm{H}(2)$ & $2270(20)$ & $11520(20)$ & $-1840(20)$ & $44(6)$ \\
\hline $\mathrm{H}(3)$ & $3220(30)$ & $9890(30)$ & $-3380(30)$ & $62(7)$ \\
\hline $\mathrm{H}(5)$ & $3740(20)$ & $7580(20)$ & $-1160(20)$ & $29(6)$ \\
\hline $\mathrm{H}(9)$ & $1670(30)$ & $12970(20)$ & $2330(30)$ & $53(7)$ \\
\hline $\mathrm{H}(10)$ & $910(30)$ & $14000(30)$ & $4200(30)$ & $63(8)$ \\
\hline $\mathrm{H}(12)$ & $840(30)$ & $10460(20)$ & $4570(30)$ & $52(7)$ \\
\hline $\mathrm{H}(13)$ & $1510(20)$ & $9400(20)$ & $2680(20)$ & $38(6)$ \\
\hline $\mathrm{H}(14 \mathrm{~A})$ & $5340(30)$ & $6830(20)$ & $-2240(30)$ & $59(7)$ \\
\hline $\mathrm{H}(14 \mathrm{~B})$ & $4730(30)$ & $6120(30)$ & $-4120(30)$ & $66(8)$ \\
\hline $\mathrm{H}(14 \mathrm{C})$ & $3500(30)$ & $5930(30)$ & $-3470(30)$ & $69(8)$ \\
\hline $\mathrm{H}(16 \mathrm{~A})$ & $2150(50)$ & $5520(50)$ & $-890(50)$ & $170(20)$ \\
\hline $\mathrm{H}(16 \mathrm{~B})$ & $1330(30)$ & $6780(30)$ & $-1010(30)$ & $64(8)$ \\
\hline $\mathrm{H}(16 \mathrm{C})$ & $940(40)$ & $5770(30)$ & $-410(30)$ & $99(11)$ \\
\hline $\mathrm{H}(18 \mathrm{~A})$ & $3700(40)$ & $8420(30)$ & $4200(30)$ & $100(11)$ \\
\hline $\mathrm{H}(18 \mathrm{~B})$ & $5300(30)$ & $8780(30)$ & $4040(30)$ & $84(10)$ \\
\hline $\mathrm{H}(18 \mathrm{C})$ & $5180(30)$ & $7590(30)$ & $4550(30)$ & $75(8)$ \\
\hline $\mathrm{H}(19 \mathrm{~A})$ & $1810(30)$ & $6180(30)$ & $2430(30)$ & $68(8)$ \\
\hline $\mathrm{H}(19 \mathrm{~B})$ & $3140(30)$ & $5430(30)$ & $2970(30)$ & $63(7)$ \\
\hline $\mathrm{H}(19 \mathrm{C})$ & $2110(30)$ & $4850(30)$ & $1130(30)$ & $70(9)$ \\
\hline $\mathrm{H}(20 \mathrm{~A})$ & $5560(40)$ & $5760(30)$ & $2600(40)$ & $102(11)$ \\
\hline $\mathrm{H}(20 \mathrm{~B})$ & $4280(30)$ & $5310(30)$ & $810(40)$ & $82(10)$ \\
\hline $\mathrm{H}(20 \mathrm{C})$ & $5620(40)$ & $6890(30)$ & $1880(30)$ & $95(11)$ \\
\hline
\end{tabular}



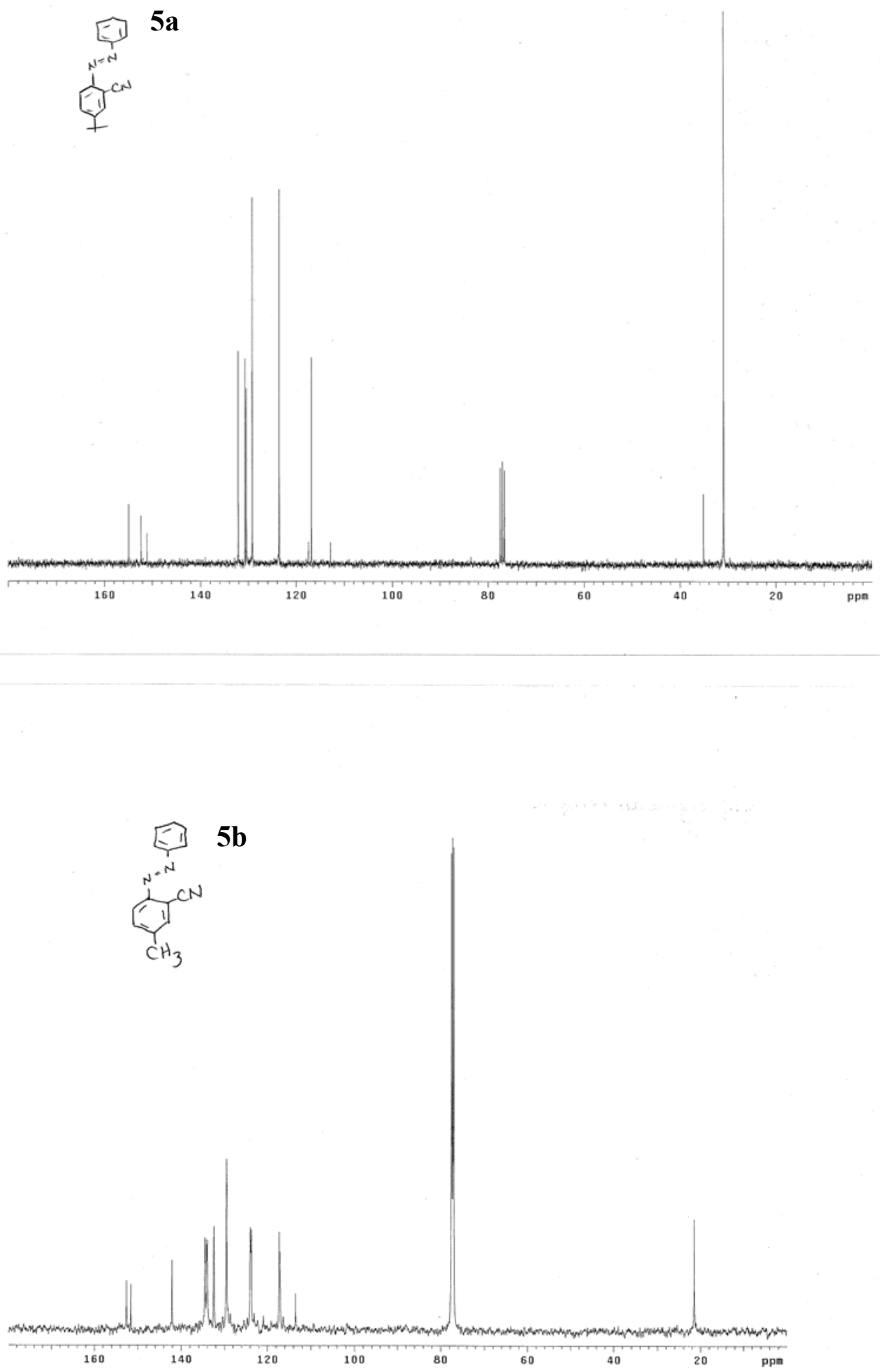

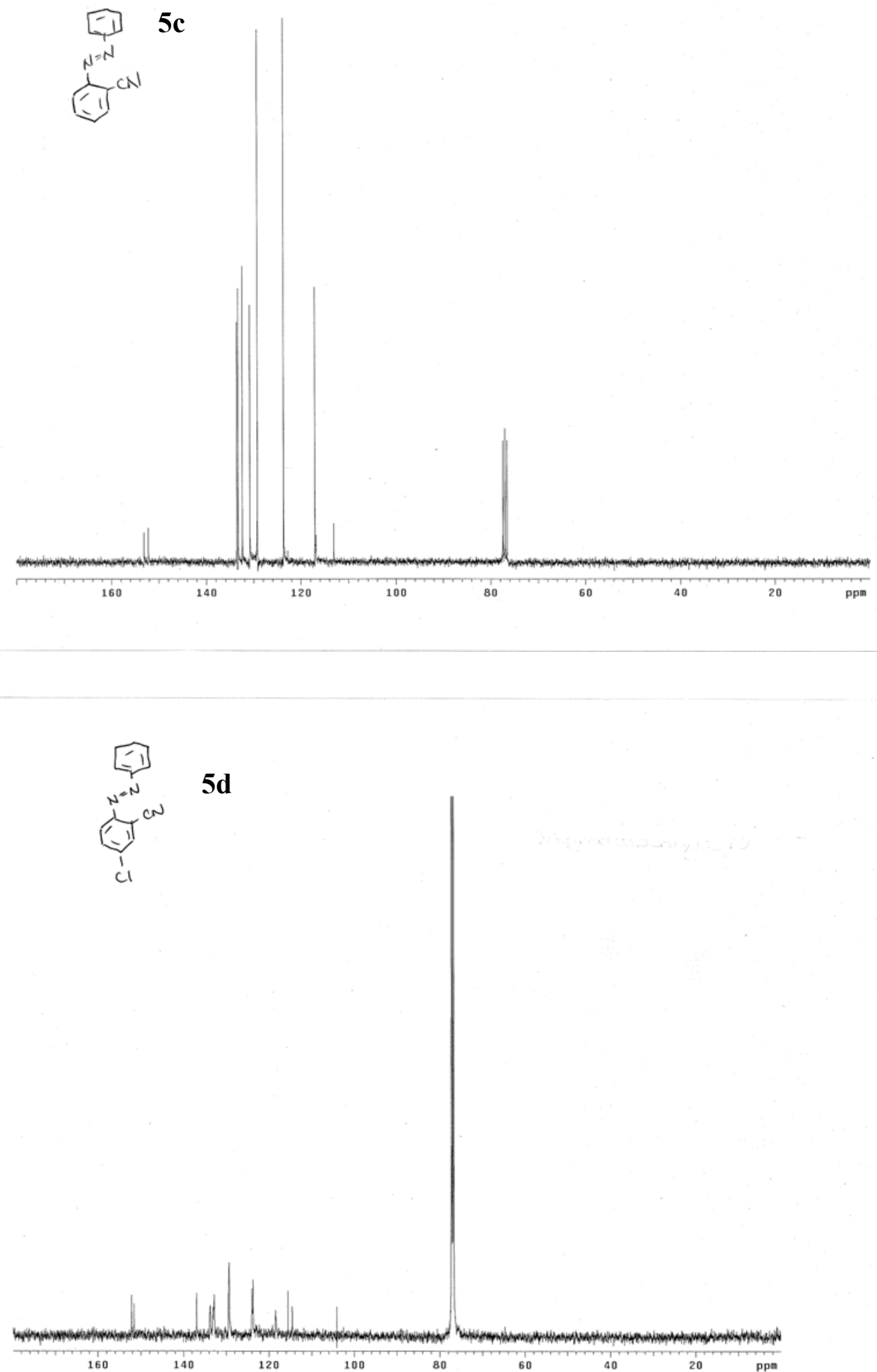

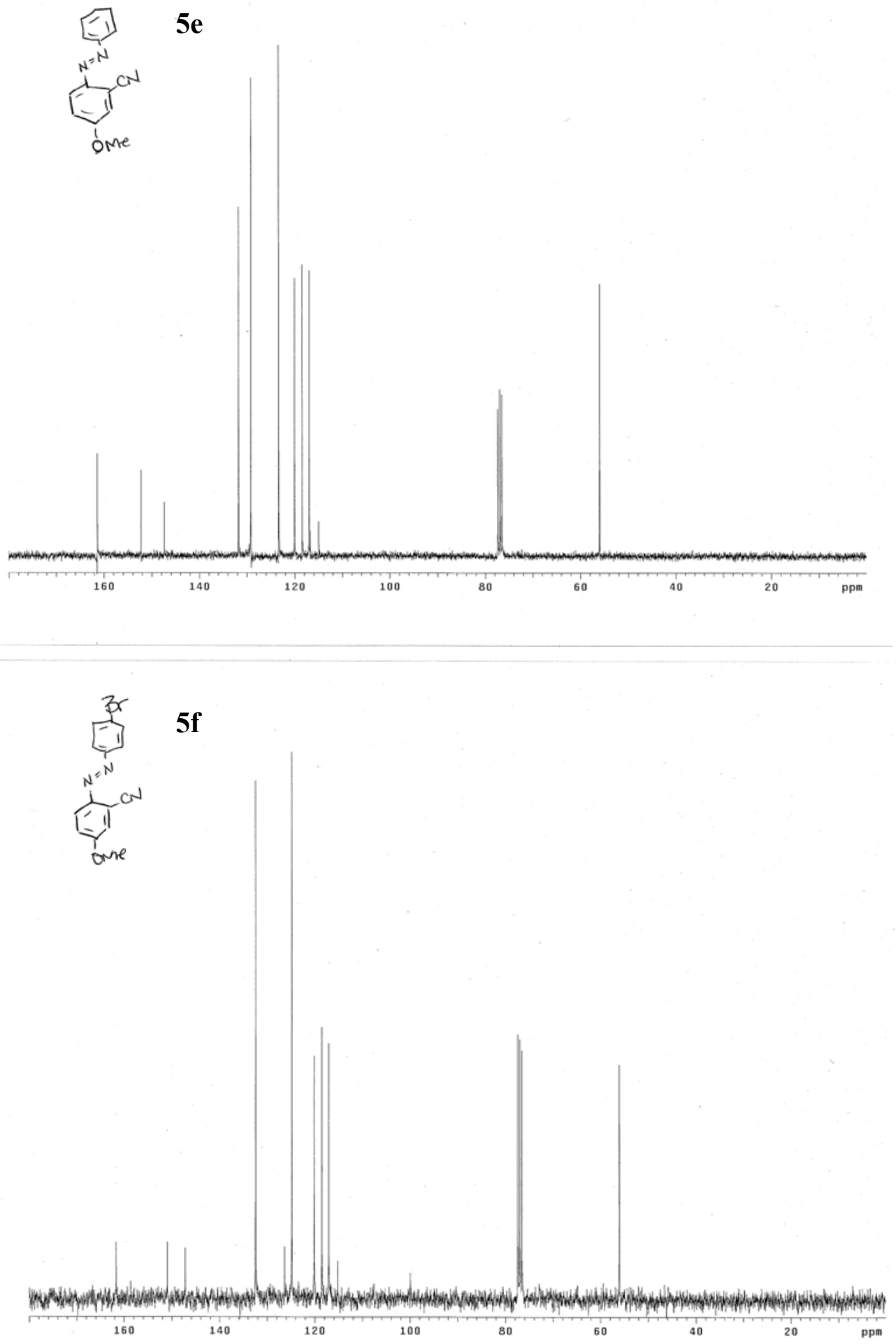

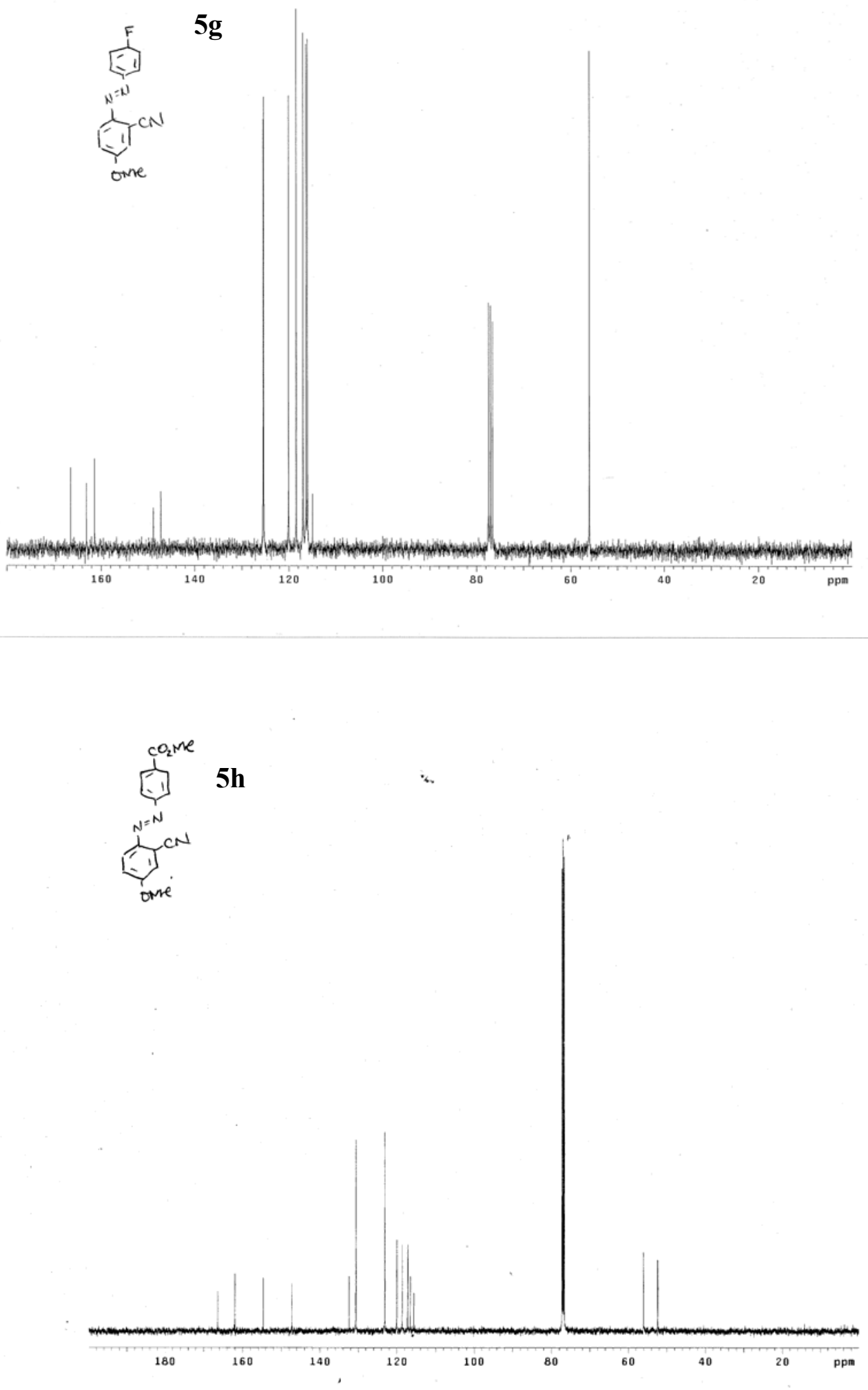

S23 

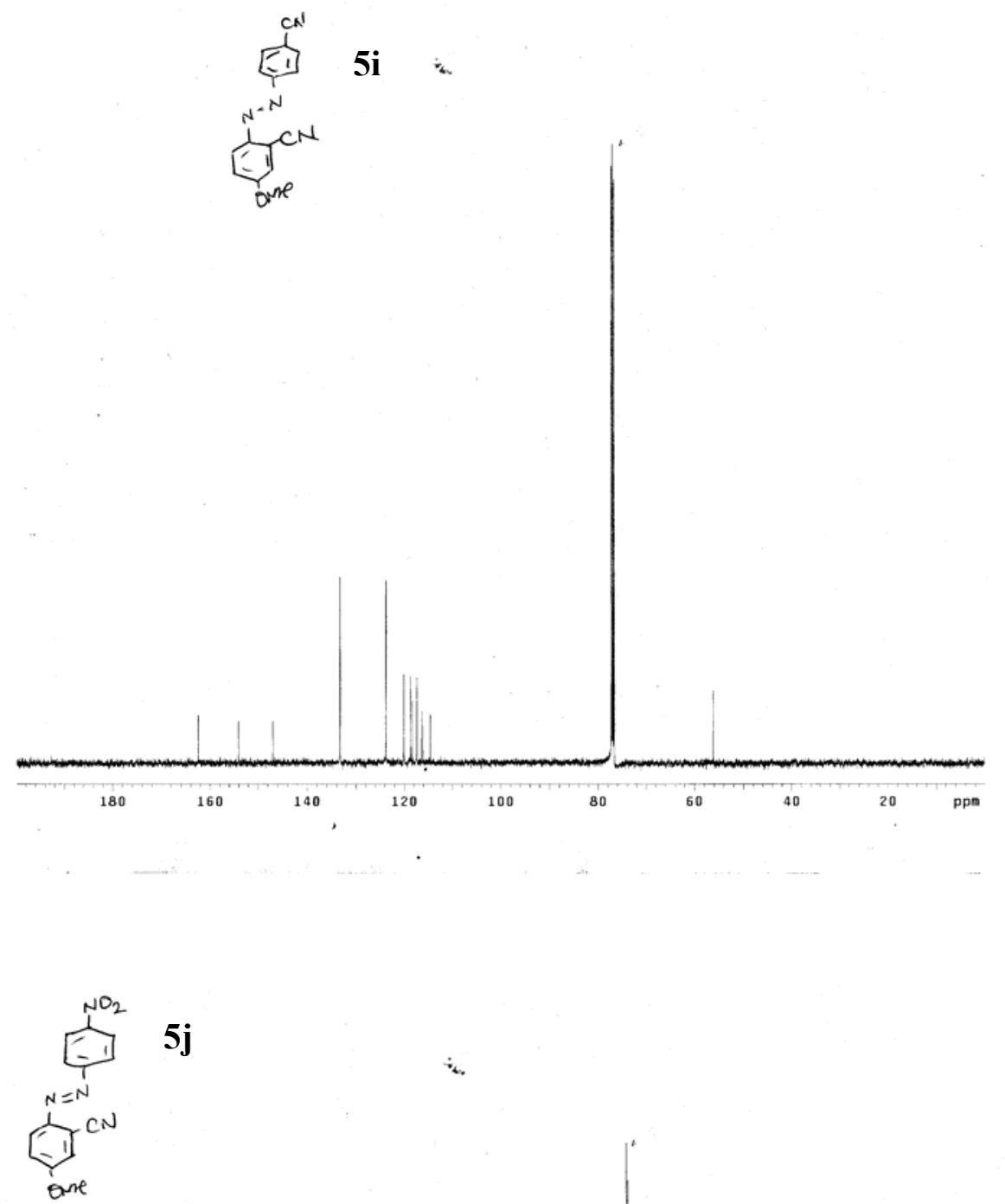

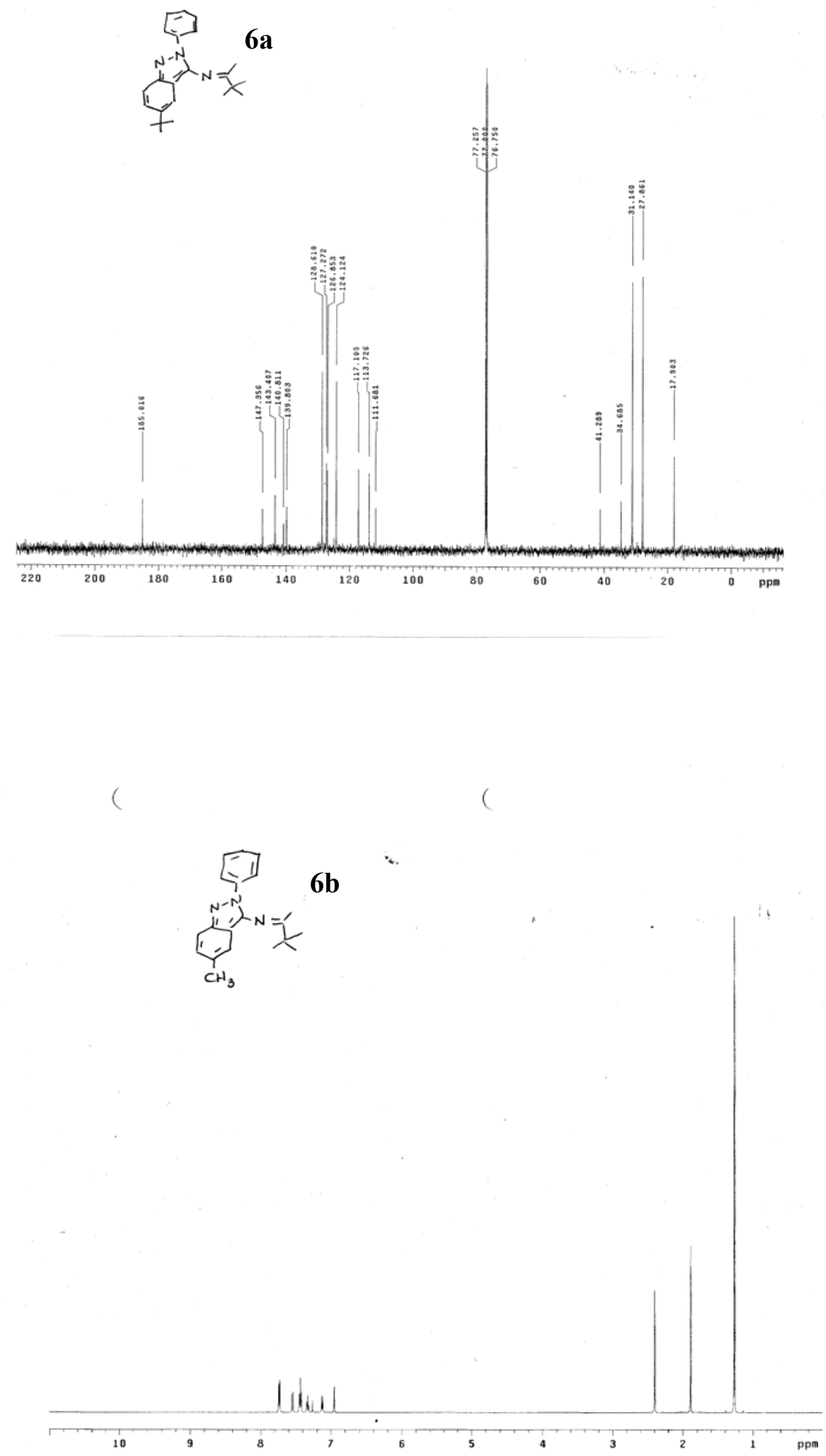

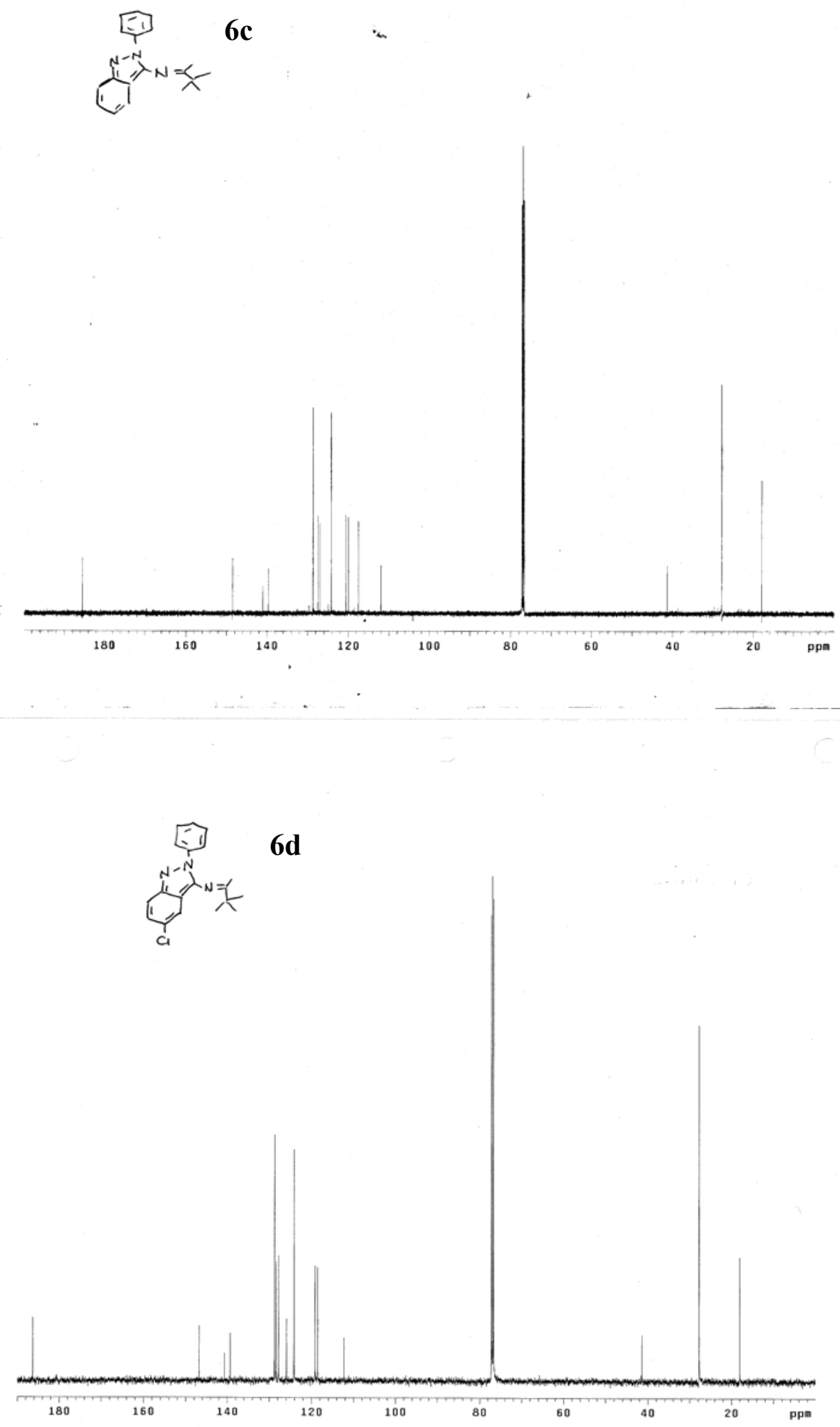

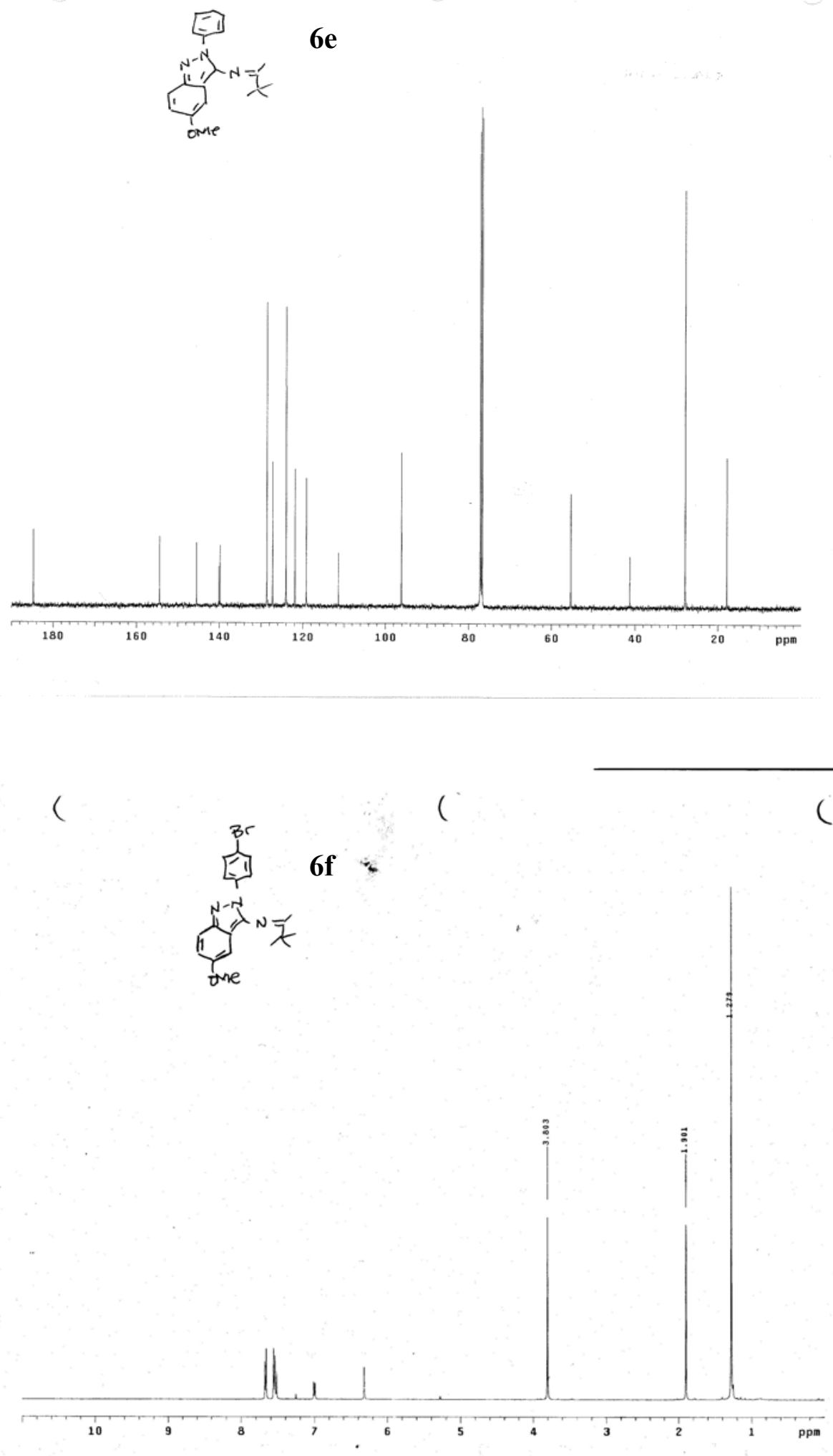

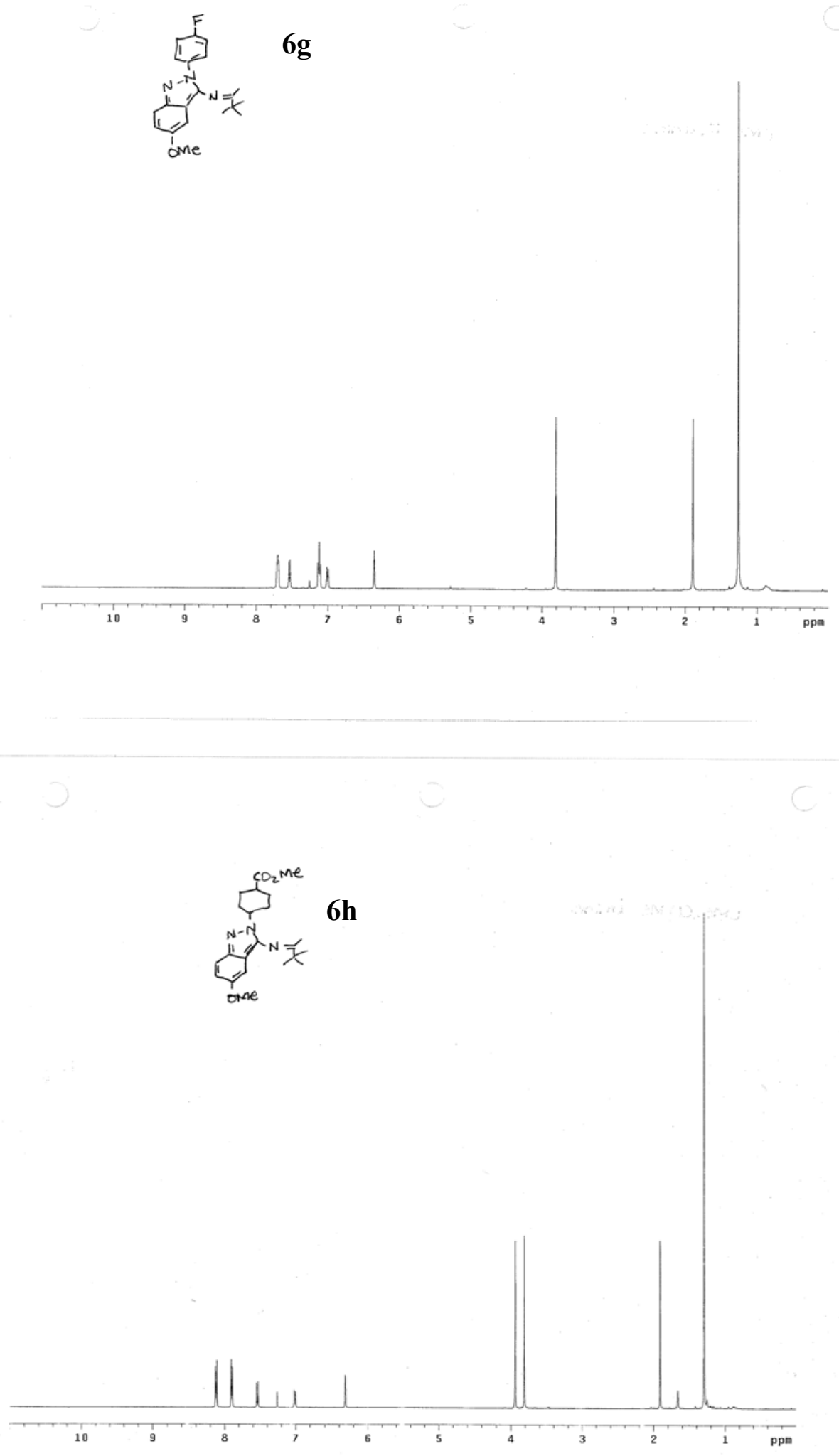

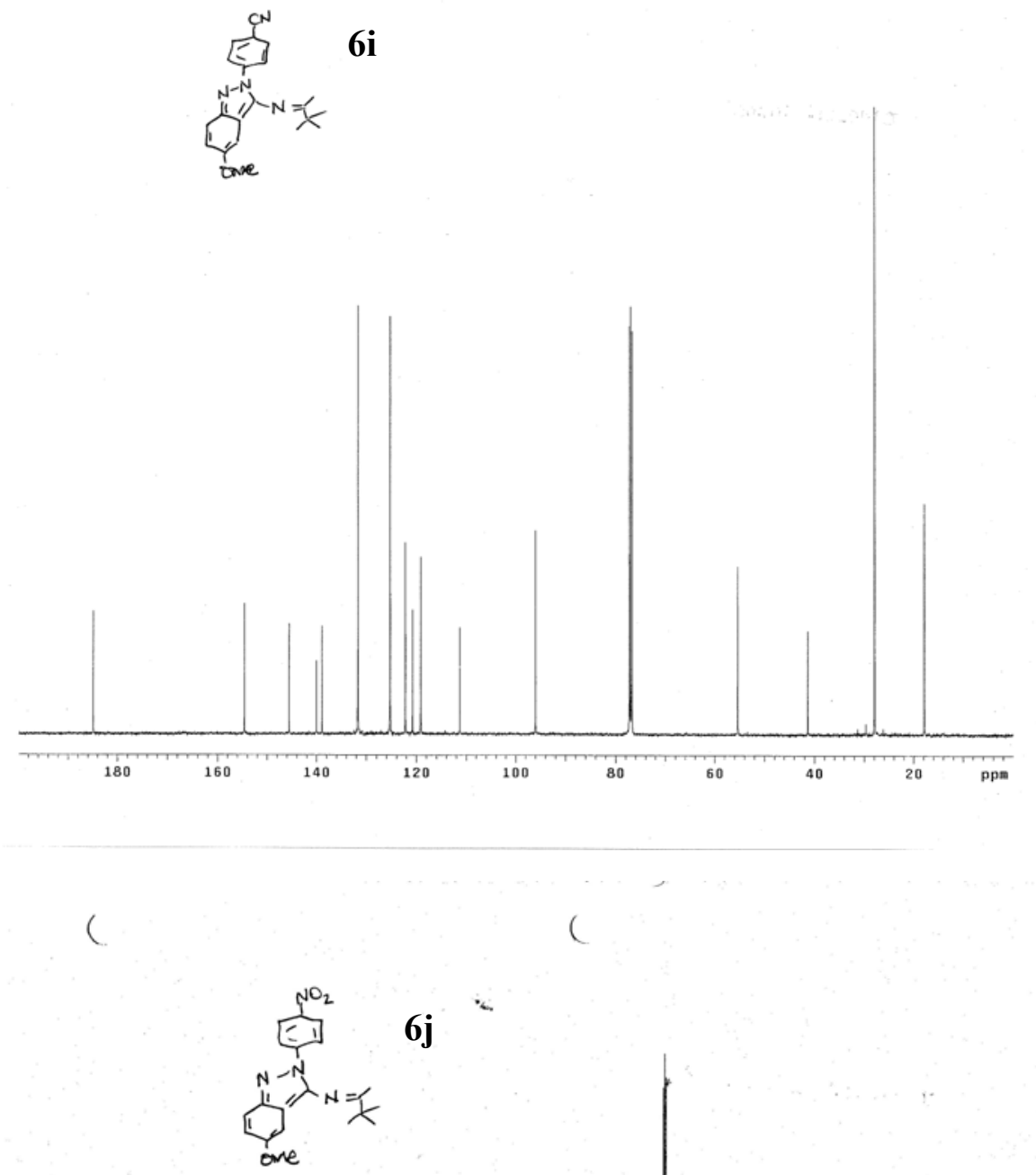
$7 a$
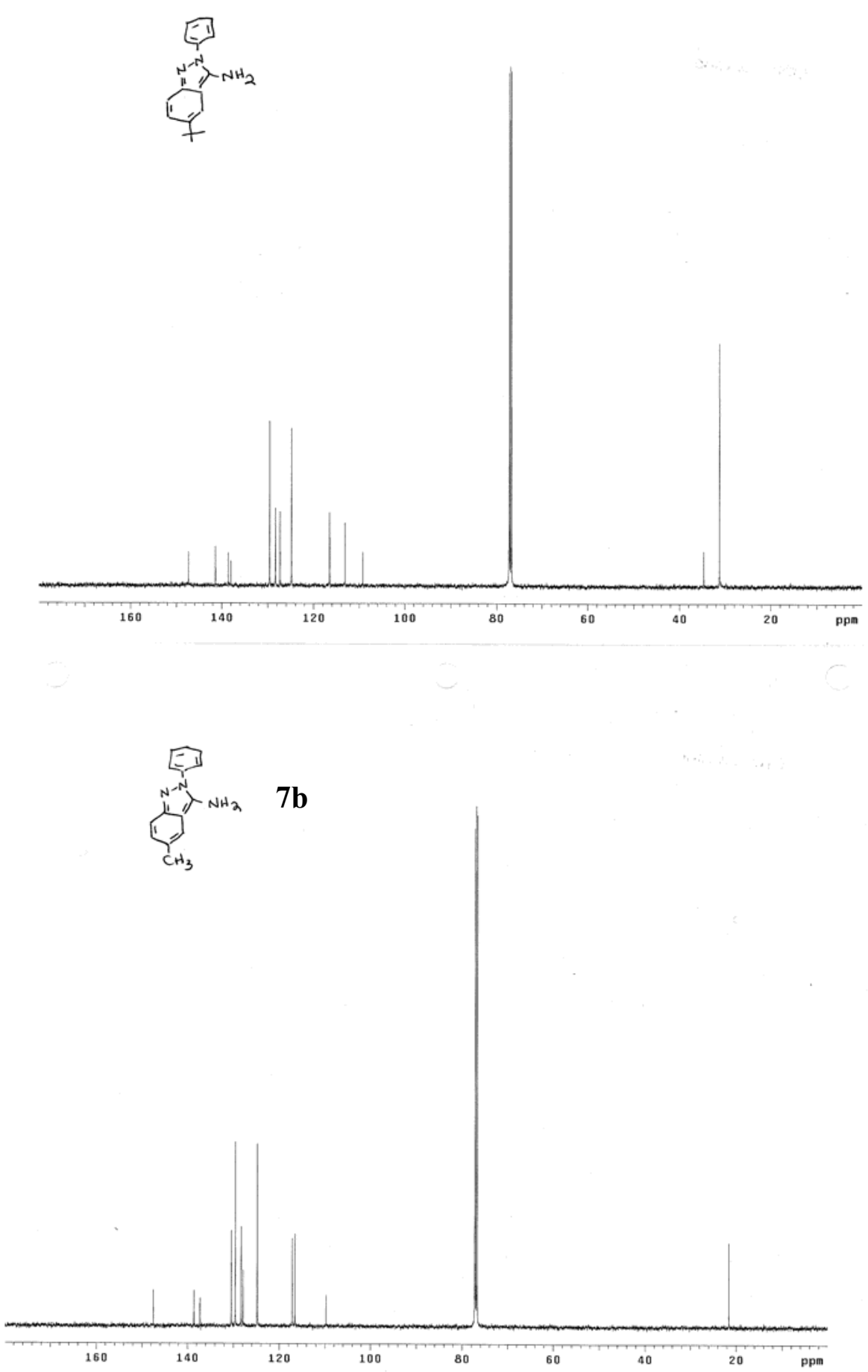


$$
\frac{11}{11}
$$



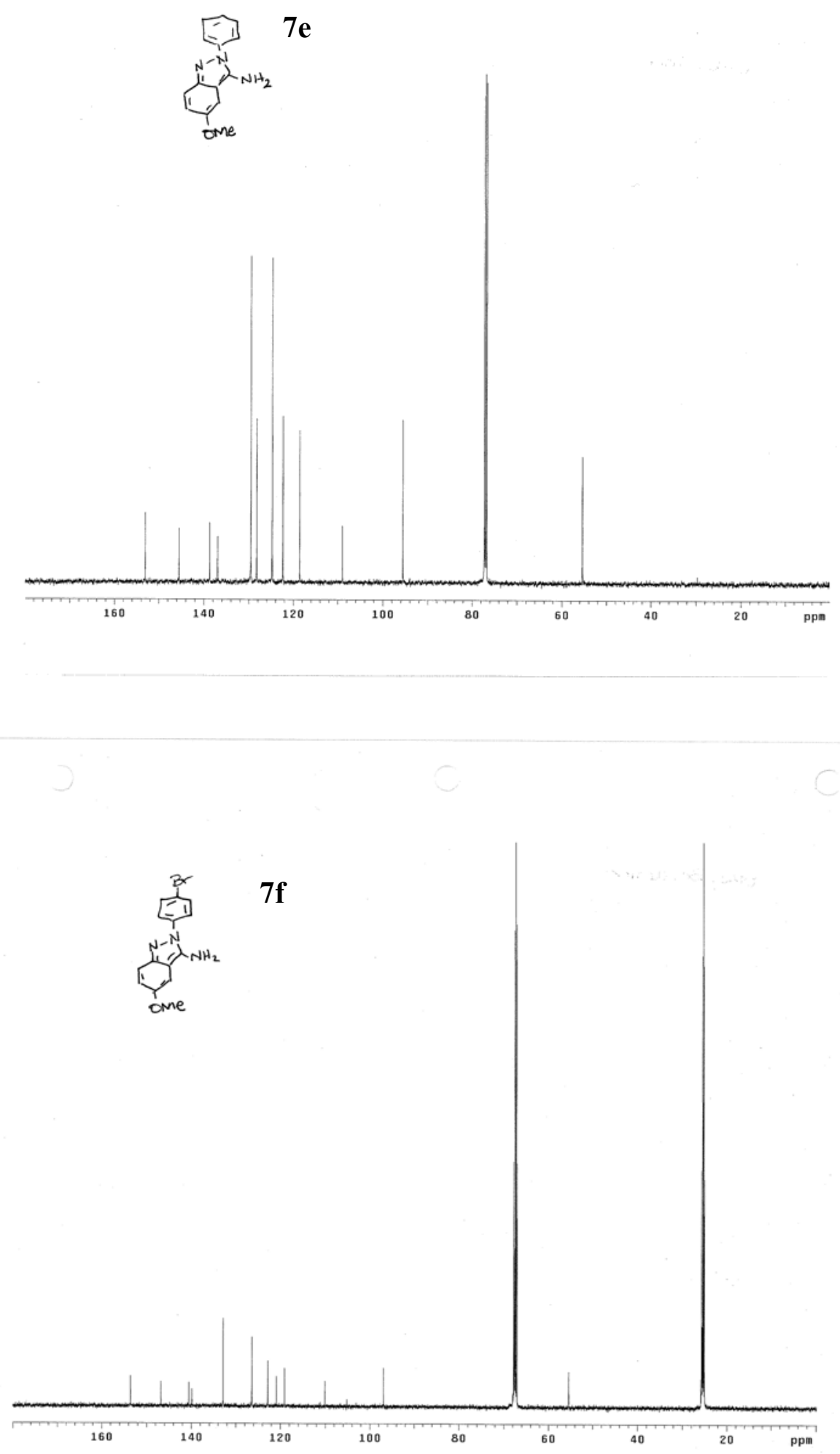

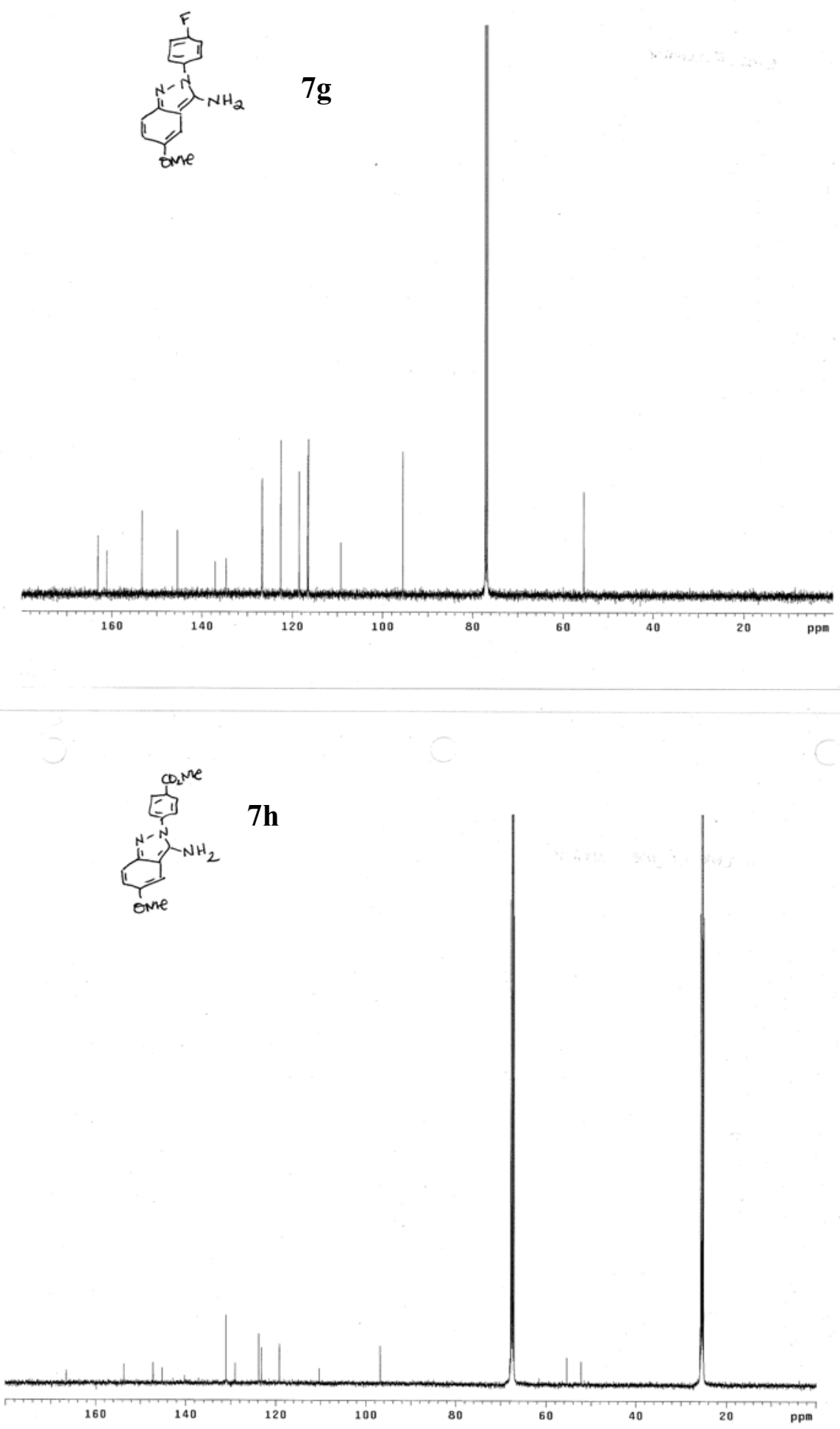

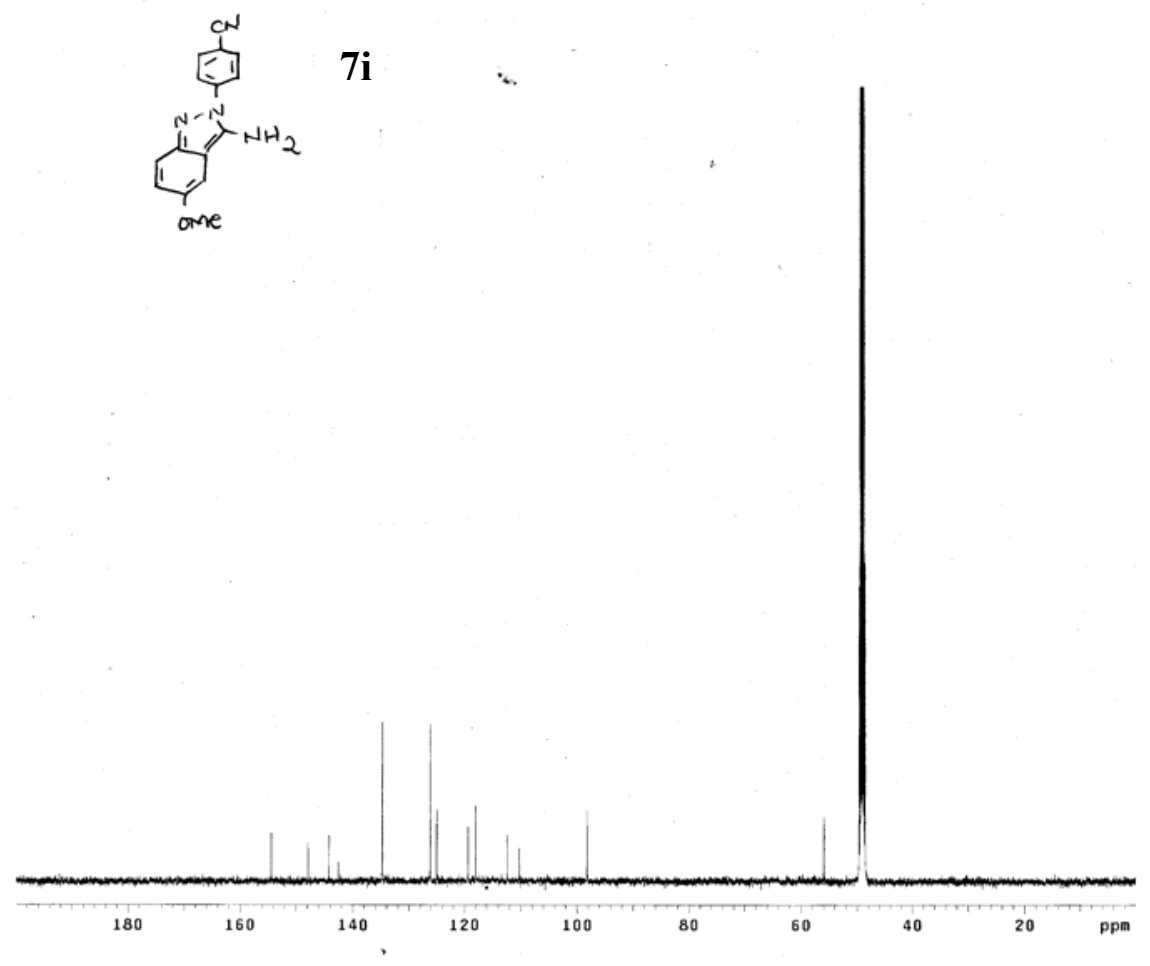

- $\quad-\quad \ldots \ldots+\ldots$
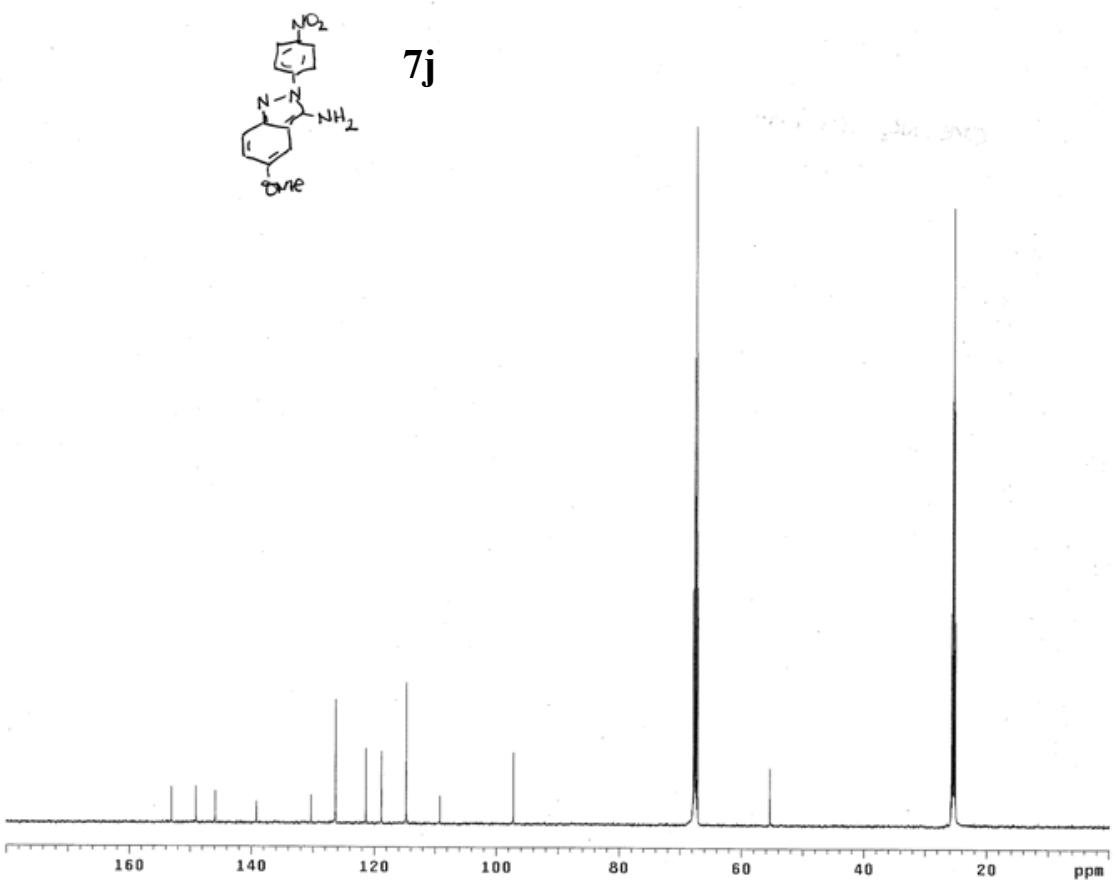